\title{
Light-front Bethe-Salpeter equation
}

\author{
J. H. O. Sales, ${ }^{1}$ T. Frederico, ${ }^{1}$ B. V. Carlson, ${ }^{1}$ and P. U. Sauer ${ }^{2}$ \\ ${ }^{1}$ Departamento de Física, Instituto Tecnológico de Aeronáutica, Centro Técnico Aeroespacial, \\ 12.228-900 São José dos Campos, São Paulo, Brazil \\ ${ }^{2}$ Institute for Theoretical Physics, University Hannover, D-30167 Hannover, Germany
}

(Received 29 July 1999; published 10 March 2000)

\begin{abstract}
A three-dimensional reduction of the two-particle Bethe-Salpeter equation is proposed. The proposed reduction is in the framework of light-front dynamics. It yields auxiliary quantities for the transition matrix and the bound state. The arising effective interaction can be perturbatively expanded according to the number of particles exchanged at a given light-front time. An example suggests that the convergence of the expansion is rapid. This result is particular for light-front dynamics. The covariant results of the Bethe-Salpeter equation can be recovered from the corresponding auxiliary three-dimensional ones. The technical procedure is developed for a two-boson case; the idea for an extension to fermions is given. The technical procedure appears quite practicable, possibly allowing one to go beyond the ladder approximation for the solution of the Bethe-Salpeter equation. The relation between the three-dimensional light-front reduction of the field-theoretic Bethe-Salpeter equation and a corresponding quantum-mechanical description is discussed.
\end{abstract}

PACS number(s): 24.85.+p, 12.39.Ki, 14.40.Cs, 13.40.Gp

\section{INTRODUCTION}

In relativistic field theory the Bethe-Salpeter equation (BSE) [1] describes two-particle systems in interaction. The inhomogeneous BSE

$$
T=V+V G_{0} T
$$

yields the transiton matrix $T$ of two-particle scattering. In Eq. (1) $G_{0}$ is the disconnected Green's function for two particles, which is reduced to the Green's function of two noninteracting particles by neglecting self-energy parts, i.e., by taking

$$
G_{0}=\frac{i}{\hat{k}_{1}^{2}-m_{1}^{2}+i o} \frac{i}{\hat{k}_{2}^{2}-m_{2}^{2}+i o},
$$

with $\hat{k}_{i}^{\mu}$ denoting the off-mass-shell momentum operator acting on the coordinates of particle $i$ with mass $m_{i}$, the hat on the variable emphasizing its operator character. The driving term $V$ stands for the complete interaction, irreducible with respect to two-particle propagation. If the dynamics allows for a two-particle bound state with total four-momentum $K_{B}$, $K_{B}^{2}=M_{B}^{2}$, the vertex $\mid \Gamma$ ) (the round ket indicates the dependence on four-dimensional coordinates of all particles) at the bound-state pole is solution of the homogeneous BSE

$$
\left.\mid \Gamma)=V G_{0} \mid \Gamma\right)
$$

with the relation

$$
\left.\mid \Psi)=G_{0} \mid \Gamma\right)
$$

to the Bethe-Salpeter amplitude $\mid \Psi)$ of the bound state. Equations (1) and (3) do not determine $\mid \Psi$ ) in full; the normalization condition has to be added. The two-particle total four-momentum $K$ is conserved in Eqs. (1) and (3); that is,
$\mathcal{O}_{\alpha}=T, G_{0}$ or $V$, as well as $\left.\mid \Psi\right)$ and $\left.\mid \Gamma\right)$ carry a fourdimensional $\delta$ function in momentum space,

$$
\begin{gathered}
\left\langle K^{\prime}\left|\mathcal{O}_{\alpha}\right| K\right\rangle=\delta\left(K^{\prime}-K\right) \mathcal{O}_{\alpha}(K), \\
\left.\left\langle K^{\prime}\right| \Psi\right)=\delta\left(K^{\prime}-K_{B}\right)\left|\Psi_{B}\right\rangle, \\
\left.\left\langle K^{\prime}\right| \Gamma\right)=\delta\left(K^{\prime}-K_{B}\right)\left|\Gamma_{B}\right\rangle,
\end{gathered}
$$

the reduced quantities depending parametrically on $K$, even if not spelled out explicitly for $\left|\Gamma_{B}\right\rangle$ and $\left|\Psi_{B}\right\rangle$. The reduced quantities $\left|\Psi_{B}\right\rangle,\left|\Gamma_{B}\right\rangle$ and the $\mathcal{O}_{\alpha}(K)$ are functions of the internal variables expressed in terms of the four-dimensional momentum $k^{\mu}$ or coordinate $x^{\mu}$. They satisfy the Eqs. (1) and (3) in a corresponding fashion. For convenience we have used the bra-ket notation to represent functions which can be written in either momentum or coordinate spaces.

The inhomogeneous and homogeneous BSEs (1) and (3) are general and exact formulations for the scattering amplitude and bound state. However, for any realistic field theory solution of the BSE constitutes a difficult calculational task which has not been tackled in full. In practical calculations, the driving term $V(K)$ has to be truncated to low orders of particle exchange. In Euclidean space, the fermion case has only been solved in ladder approximation [2], i.e., with single particle exchange for the driving term, while the boson case has only been solved in ladder and crossed ladder approximation [3]. However, the step from the Euclideanspace to Minkowski-space solutions requires a complicated analytic continuation [4]. Direct solutions in Minkowski space are just now becoming available [5].

In the light of the great calculational difficulties, threedimensional reductions of the BSE are still of high physics interest. The conceptual sacrifices generated by the reduction can possibly be outweighed by the gain in technical ease. One hopes to be able to include physical phenomena which the four-dimensional BSE with a highly truncated interaction is unable to account for. For example, the three-dimensional 
Gross approach [6] allows only one particle to propagate off-mass-shell, but it appears to go beyond the ladder approximation of BSE by single particle exchange and to include crossed exchanges implicitly; it manfestly preserves covariance. Other reduction schemes give up covariance, which then must be recovered through complicated correction schemes. An equal-time projection scheme has also been explored for the pion-nucleon system which fulfills requirements of covariance and discrete Poincarè symmetries [7]. The papers by Fuda [8] report on the comparision of onemeson exchange models in ladder approximation on both light-front and instant-form dynamics, without emphasis to the underlying field-theoretic framework. The field theoretic approach in the light front has also been recently used with success to describe finite nuclei [9] and nuclear matter with nucleon-nucleon correlation [10]. After the submission of this work, Ref. [11] came to our attention, which discusses the light-front description of the angular momentum bound states of the Bethe-Salpeter equation for the same bosonic model used here.

The purpose of this paper is twofold. (i) First, the paper attempts to find a three-dimensional equation for auxiliary quantities from which the full covariant solution of the BSE in the ladder or any other approximation can be obtained with ease. This is a technical objective with solutions wellknown in the framework of instant-form dynamics. Here the advantages of light-front dynamics are to be explored.

(ii) Second, the paper tries to illuminate the connection to a quantum-mechanical description of the two-particle system whose dynamic input is related to the underlying field theory.

Section II motivates our novel choice for threedimensional auxiliary quantities from which the covariant solutions of the BSE are obtained. It motivates light-front dynamics as our choice for a dynamical framework. Section III gives our theoretical apparatus in full. Section IV tests the potential of the method in the example of a two-boson bound state. We perform numerical calculations for the two-boson bound state including up to four-particle intermediate states in lowest order and compare to the solutions of the fourdimensional BSE equation in the ladder approximation. Section $\mathrm{V}$ sketches the generalization of our theoretical apparatus to fermions. Section VI discusses the connection with light-front quantum mechanics. Our conclusions are summarized in Sec. VII.

\section{CHOICE OF TWO-PARTICLE AUXILIARY FREE GREEN'S FUNCTION $\widetilde{G}_{0}(K)$}

It is well known, from the work of Ref. [12], that the transition matrix $T(K)$ and the Bethe-Salpeter amplitude $\left|\Psi_{B}\right\rangle$ of the covariant BSE can be obtained with the help of a convenient auxiliary Green's function $\widetilde{G}_{0}(K)$, still to be chosen. That is, we have

$$
\begin{gathered}
T(K)=W(K)+W(K) \widetilde{G}_{0}(K) T(K), \\
\left|\Gamma_{B}\right\rangle=W\left(K_{B}\right) \widetilde{G}_{0}\left(K_{B}\right)\left|\Gamma_{B}\right\rangle,
\end{gathered}
$$

$$
\left|\Psi_{B}\right\rangle=G_{0}(K)\left|\Gamma_{B}\right\rangle,
$$

provided the driving term $V(K)$ is changed to $W(K)$ according to

$$
W(K)=V(K)+V(K)\left[G_{0}(K)-\widetilde{G}_{0}(K)\right] W(K) .
$$

Equations (9) and (10) do not determine $\left|\Psi_{B}\right\rangle$ in full; the normalization condition

$$
\begin{aligned}
& \lim _{K^{2} \rightarrow K_{B}^{2}}\left\langle\Psi_{B}\left|\frac{G_{0}(K)^{-1}-G_{0}\left(K_{B}\right)^{-1}}{K^{2}-K_{B}^{2}}-\frac{V(K)-V\left(K_{B}\right)}{K^{2}-K_{B}^{2}}\right| \Psi_{B}\right\rangle \\
& \quad=1
\end{aligned}
$$

has to be added. It involves the original driving term $V(K)$ [13]. The choice of $\widetilde{G}_{0}(K)$ is hoped to be sufficently clever that the integral equation (11) does not have to be solved in full, but that a few terms of the infinite series

$$
\begin{gathered}
W(K)=V(K) \sum_{n=0}^{\infty}\left[\left(G_{0}(K)-\widetilde{G}_{0}(K)\right) V(K)\right]^{n}, \\
W(K)=V(K)+V(K)\left(G_{0}(K)-\widetilde{G}_{0}(K)\right) V(K)+\cdots
\end{gathered}
$$

suffice. The auxiliary Green's function $\widetilde{G}_{0}(K)$ remains a four-dimensional one, but its choice may sacrifice the covariance which $G_{0}(K)$ possesses.

The dynamics of the interacting two-particle system can be fully described by its propagation between hyperplanes, the hyperplanes $x^{0}=$ const in instant-form dynamics, the hyperplanes $x^{+}=x^{0}+x^{3}=$ const in light-front dynamics [14]. Among the hyperplanes of $x^{+}=$const, only the light front with $x^{+}=0$ is left invariant by seven kinematical boosts, while the hyperplane $x^{+}=$const scales under light-front boosts. In contrast, the free Green's function of the BSE depends on the individual times $x_{i}^{0}$ or on the individual lightfront times $x_{i}^{+}$.

The free Green's function in instant-form coordinates, e.g., $k_{i}=\left(k_{i}^{0}, \vec{k}_{i}\right)$

$$
\begin{aligned}
\left\langle x_{1}^{\prime 0}{ }_{1}{ }^{\prime}{ }_{2}\left|G_{0}\right| x_{1}^{0} x_{2}^{0}\right\rangle= & -\frac{1}{(2 \pi)^{2}} \int d k_{1}^{0} d K^{0} \\
& \times \frac{e^{-i k_{1}^{0}\left(x^{\prime}{ }_{1}^{0}-x^{\prime}{ }_{2}-x_{1}^{0}+x_{2}^{0}\right)}}{\left[\left(k_{1}^{0}\right)^{2}-\hat{\vec{k}}_{1}^{2}-m_{1}^{2}+i o\right]} \\
& \times \frac{e^{-i K^{0}\left(x^{\prime}{ }_{2}-x_{2}^{0}\right)}}{\left[\left(K^{0}-k_{1}^{0}\right)^{2}-\left(\vec{K}-\hat{\vec{k}}_{1}\right)^{2}-m_{2}^{2}+i o\right]}
\end{aligned}
$$

-in fact only its dependence on individual times $x_{i}^{0}$ is made explicit-reduces for propagation between the hyperplanes $x_{1}^{0}=x_{2}^{0}=x^{0}$ and $x_{1}^{\prime 0}=x_{2}^{\prime 0}=x^{\prime 0}$ to 


$$
\begin{aligned}
\left\langle x^{\prime 0} x^{\prime 0}\left|G_{0}\right| x^{0} x^{0}\right\rangle & =\int \frac{d K^{0}}{2 \pi} e^{-i K^{0}\left(x^{\prime 0}-x^{0}\right)} \int d k_{1}^{\prime 0} d k_{1}^{0}\left\langle k^{\prime 0}\left|G_{0}(K)\right| k_{1}^{0}\right\rangle, \\
& \left.\left.\equiv \int \frac{d K^{0}}{2 \pi} e^{-i K^{0}\left(x^{\prime 0}-x^{0}\right)}\right|_{0} G_{0}(K)\right|_{0} .
\end{aligned}
$$

In Eq. (15) the notation

$$
\left\langle k^{\prime \prime}{ }_{1}^{0}\left|G_{0}(K)\right| k_{1}^{0}\right\rangle=-\frac{1}{2 \pi} \frac{\delta\left(k_{1}^{\prime 0}-k_{1}^{0}\right)}{\left[\left(k_{1}^{0}\right)^{2}-\hat{\vec{k}}_{1}^{2}-m_{1}^{2}+i o\right]\left[\left(K^{0}-k_{1}^{0}\right)^{2}-\left(\vec{K}-\hat{\vec{k}}_{1}\right)^{2}-m_{2}^{2}+i o\right]}
$$

is introduced, as well as the abbreviation

$$
\begin{aligned}
\left|{ }_{0} G_{0}(K)\right|_{0} & :=\int d k_{1}^{\prime 0} d k_{1}^{0}\left\langle k_{1}^{\prime 0}\left|G_{0}(K)\right| k_{1}^{0}\right\rangle \\
& =\frac{i}{2 \hat{k}_{1 o n}^{0} 2 \hat{k}_{2 o n}^{0}}\left(\frac{1}{\left(K^{0}-\hat{k}_{1 o n}^{0}-\hat{k}_{2 o n}^{0}+i o\right)}-\frac{1}{\left(K^{0}+\hat{k}_{1 o n}^{0}+\hat{k}_{2 o n}^{0}-i o\right)}\right) .
\end{aligned}
$$

The matrix element $\left\langle k_{1}^{\prime 0}\left|G_{0}(K)\right| k_{1}^{0}\right\rangle$ of Eq. (17), in which only the dependence on the "dynamic'" variable $k_{1}^{0}$ is made explicit remains an operator with respect to the "kinematic"' variables $\vec{k}_{1}$, the operator character being carried by the operators $\hat{k}_{i o n}^{0}$ $=\sqrt{\hat{\vec{k}}_{i}^{2}+m_{i}^{2}}$ acting on functions of kinematic variables. The basis states for functions of these kinematic variables are defined by $\left\langle\vec{x}_{i} \mid \vec{k}_{i}\right\rangle=\exp \left(\imath \vec{k}_{i} \cdot \vec{x}_{i}\right)$ and are eigenfunctions of the momentum operator $\hat{\vec{k}}$ and the free energy operator $\hat{k}_{\text {on }}^{0}$. The states $|\vec{k}\rangle$ form an orthogonal and complete basis for functions of the kinematic variables.

In Eq. (18), the vertical bar $\left.\right|_{0}$ indicates that the dependence on $k_{1}^{0}$ is integrated out. The bar on the left of the Green's function represents integration on $k_{1}^{0}$ in the bra state, the bar on the right in the ket state; we shall encounter Green's functions in which integration on $k_{1}^{0}$ is performed only on one side, the bar $\left.\right|_{0}$ being placed on that side alone. The resulting function $\left.{ }_{0} G_{0}(K)\right|_{0}$ is three dimensional and depends only on the kinematic variables $\vec{k}_{1}$. It is a global propagator, since it mediates between hyperplanes according to Eq. (16), not allowing for individual time differences between the two particles, it is not explicitly covariant. In instant-form dynamics, the global propagator $\left.\left.\right|_{0} G_{0}(K)\right|_{0}$ still allows for particle and antiparticle propagation. This is considered to be a technical disadvantage.

The free Green's function in light-front coordinates, e.g., $k_{i}=\left(k_{i}^{-}:=k_{i}^{0}-k_{i}^{3}, \quad k_{i}^{+}:=k_{i}^{0}+k_{i}^{3}, \quad \vec{k}_{i \perp}\right)$

$$
\begin{aligned}
\left\langle x_{1}^{\prime+} x_{2}^{\prime+}\left|G_{0}\right| x_{1}^{+} x_{2}^{+}\right\rangle= & -\frac{1}{(2 \pi)^{2}} \int d k_{1}^{-} d K^{-} e^{-i / 2 k_{1}^{-}\left(x_{1}^{\prime+}-x_{2}^{\prime+}-x_{1}^{+}+x_{2}^{+}\right)} e^{-i / 2 K^{-}\left(x_{2}^{\prime+}-x_{2}^{+}\right)} \\
& \times \frac{1}{\hat{k}_{1}^{+}\left(K^{+}-\hat{k}_{1}^{+}\right)\left(k_{1}^{-}-\frac{\hat{\vec{k}}_{1 \perp}^{2}+m_{1}^{2}-i o}{\hat{k}_{1}^{+}}\right)\left(K^{-}-k_{1}^{-}-\frac{\hat{\vec{k}}_{2 \perp}^{2}+m_{2}^{2}-i o}{K^{+}-\hat{k}_{1}^{+}}\right)}
\end{aligned}
$$

-only its dependence on the individual light-front "times" $x_{i}^{+}$is made explicit—reduces, for propagation between the hyperplanes $x_{1}^{+}=x_{2}^{+}=x^{+}$and $x_{1}^{\prime+}=x_{2}^{\prime+}=x^{\prime+}$, to

$$
\begin{aligned}
\left\langle x^{\prime+} x^{\prime+}\left|G_{0}\right| x^{+} x^{+}\right\rangle & =\int \frac{d K^{-}}{2 \pi} e^{-i / 2 K^{-}\left(x^{\prime+}-x^{+}\right)} \int d k_{1}^{\prime-} d k_{1}^{-}\left\langle k_{1}^{\prime-}\left|G_{0}(K)\right| k_{1}^{-}\right\rangle, \\
& \equiv \int \frac{d K^{-}}{2 \pi} e^{-i / 2 K^{-}\left(x^{\prime+}-x^{+}\right)}\left|G_{0}(K)\right| .
\end{aligned}
$$

In Eq. (21) the notation 


$$
\left\langle k_{1}^{\prime-}\left|G_{0}(K)\right| k_{1}^{-}\right\rangle=-\frac{1}{2 \pi} \frac{\delta\left(k_{1}^{\prime-}-k_{1}^{-}\right)}{\hat{k}_{1}^{+}\left(K^{+}-\hat{k}_{1}^{+}\right)\left(k_{1}^{-}-\frac{\hat{\vec{k}}_{1 \perp}^{2}+m_{1}^{2}-i o}{\hat{k}_{1}^{+}}\right)\left(K^{-}-k_{1}^{-}-\frac{\hat{\vec{k}}_{2 \perp}^{2}+m_{2}^{2}-i o}{K^{+}-\hat{k}_{1}^{+}}\right)}
$$

is introduced with the abbreviation

$$
\begin{aligned}
\left|G_{0}(K)\right|: & =\int d k_{1}^{\prime-} d k_{1}^{-}\left\langle k_{1}^{\prime-}\left|G_{0}(K)\right| k_{1}^{-}\right\rangle \\
& =\frac{i \theta\left(K^{+}-\hat{k}_{1}^{+}\right) \theta\left(\hat{k}_{1}^{+}\right)}{\hat{k}_{1}^{+}\left(K^{+}-\hat{k}_{1}^{+}\right)\left(K^{-}-\hat{k}_{1 o n}^{-}-\hat{k}_{2 o n}^{-}+i o\right)} \\
& :=g_{0}(K),
\end{aligned}
$$

where $K^{+}>0$ can be chosen without any loss of generality. However, there is a difference between $K^{+} \geqslant 0$ and $K^{+}$ $>0$. In principle $G_{0}$ can have contributions of the form $\delta\left(k^{+}\right) / k^{+}$which are related to zero modes. Their contribution could appear as a nontrivial weight at $k^{+}=0$ and are also related to the renormalization of the quantum field theory on the light front [15]. The matrix element $\left\langle k_{1}^{\prime}\left|G_{0}(K)\right| k_{1}^{-}\right\rangle$of Eq. (23), in which only the dependence on the "dynamic" variable $k_{1}^{-}$is made explicit, still remains a functional operator with respect to functions of the "kinematic", variables

$$
\left(\hat{k}_{1}^{+}, \hat{k}_{1 \perp}\right), \hat{k}_{1 \text { on }}^{-}=\frac{\hat{\vec{k}}_{1 \perp}^{2}+m_{1}^{2}}{\hat{k}_{1}^{+}}
$$

and

$$
\hat{k}_{2 o n}^{-}=\frac{\left(\vec{K}_{\perp}-\hat{\vec{k}}_{1 \perp}\right)^{2}+m_{2}^{2}}{K^{+}-\hat{k}_{1}^{+}} .
$$

The basis states for functions of the kinematical light-front variables are defined by

$$
\left\langle x_{i}^{-} \vec{x}_{i \perp} \mid k_{i}^{+} \vec{k}_{i \perp}\right\rangle=e^{-\imath\left(1 / 2 k_{i}^{+} x_{i}^{-}-\vec{k}_{i \perp} \cdot \vec{x}_{i \perp}\right)}
$$

and are eigenfunctions of the momentum operators $\left(\hat{k}_{i}^{+}, \hat{\vec{k}}_{i \perp}\right)$ and the free energy operator $\hat{k}_{i o n}^{-}$acting on functions of the kinematical variables. The states $\left|k^{+} \vec{k}_{\perp}\right\rangle$ form an orthonormal and complete basis in the space of functions of the kinematical variables, e.g.,

$$
\begin{gathered}
\int \frac{d k^{+} d^{2} k_{\perp}}{2(2 \pi)^{3}}\left\langle x^{\prime-} \vec{x}_{\perp}^{\prime} \mid k^{+} \vec{k}_{\perp}\right\rangle\left\langle k^{+} \vec{k}_{\perp} \mid x^{-} \vec{x}_{\perp}\right\rangle \\
=\delta\left(x^{\prime-}-x^{-}\right) \delta\left(\vec{x}_{\perp}^{\prime}-\vec{x}_{\perp}\right) .
\end{gathered}
$$

In Eq. (24) the vertical bar $\mid$ indicates that the dependence on $k_{1}^{-}$is integrated out. The bar on the left of the Green's function represents integration on $k_{1}^{-}$in the bra state, the bar on the right in the ket state. We shall encounter Green's functions in which integration on $k_{1}^{-}$is done on one side alone, the bar $\mid$ being placed only on that side. The operator $g_{0}(K)$ is three dimensional and it depends on the kinematic variables $\left(k_{1}^{+}, \vec{k}_{1 \perp}\right)$ only. It is a global propagator, since it mediates between hyperplanes according to Eq. (22), not allowing for individual light-front time differences between the two particles. It does not possess explicit covariance but is still covariant under light-front boosts. In light-front dynamics, the global propagator $g_{0}(K)$ only allows particle propagation, no antiparticle propagation, due to the choice of $K^{+}>0$. This is the advantage of light-front dynamics, with which we work from now on.

The auxiliary four-dimensional Green's function $\widetilde{G}_{0}(K)$, introduced in Eqs. (8)-(13) has to be chosen next. We require for $\widetilde{G}_{0}(K)$ :

$$
\begin{gathered}
\widetilde{G}_{0}(K)\left|=G_{0}(K)\right|, \\
\left|\widetilde{G}_{0}(K)=\right| G_{0}(K), \\
\left|\widetilde{G}_{0}(K)\right|=\left|G_{0}(K)\right|,
\end{gathered}
$$

and define a three-dimensional transition matrix $t(K)$ through

$$
\begin{aligned}
& \left|\left[\widetilde{G}_{0}(K)+\widetilde{G}_{0}(K) T(K) \widetilde{G}_{0}(K)\right]\right| \\
& \quad=g_{0}(K)+g_{0}(K) t(K) g_{0}(K) .
\end{aligned}
$$

In Eqs. (29)-(32) the abbreviation | for integrating out the $k_{1}^{-}$dependence of operators is used. The conditions (29)(32) are a rather mixed bag. The conditions (31) and (32) are physical ones: They require that the global-propagator form of $\widetilde{G}_{0}(K)$ be the same as for the exact free Green's function $G_{0}(K)$ and that the full Green's function of BSE $G_{0}(K)$ $+G_{0}(K) T(K) G_{0}(K)$ can be obtained from $\left|\widetilde{G}_{0}(K)\right|$ and the three-dimensional $t(K)$. However, the two conditions (31) and (32) do not determine $\widetilde{G}_{0}(K)$ in full. Our choice is

$$
\widetilde{G}_{0}(K):=G_{0}(K)\left|g_{0}^{-1}(K)\right| G_{0}(K),
$$

though $\widetilde{G}_{0}(K)=\delta\left(\hat{k}_{1}^{\prime-}-K^{-} / 2\right) g_{0}(K) \delta\left(\hat{k}_{1}^{-}-K^{-} / 2\right)$ (and obvious variants of it) seems to be a legitimate alternative. However, the conditions (29) and (30) introduce the additional convenience that the auxiliary Green's function be as close as possible to the exact free one and Eq. (33) allows the light-front propagators in higher Fock-states to appear explicitly in the kernel of integral equation for the auxiliary 
transition matrix, which will be defined in the next section. The auxiliary quantities are computed in Appendix A.

\section{CALCULATIONAL PROCEDURE}

Our calculational procedure amounts to solving threedimensional integral equations, whose solutions then yield the covariant results of the BSE by quadrature. The fourdimensional transition matrix $T(K)$ is obtained from the three-dimensional auxiliary one $t(K)$, defined by Eq. (32), through

$$
t(K)=g_{0}(K)^{-1}\left|G_{0}(K) T(K) G_{0}(K)\right| g_{0}(K)^{-1},
$$

by first iterating the integral equation (8) once,

$$
T(K)=W(K)+W(K)\left[\widetilde{G}_{0}(K)+\widetilde{G}_{0}(K) T(K) \widetilde{G}_{0}(K)\right] W(K),
$$

and then making use of our choice, Eq. (33), for $\widetilde{G}_{0}(K)$ and the result Eq. (34). The relation between the $T(K)$ and the auxiliary $t(K)$ is

$$
\begin{aligned}
T(K)= & W(K)+W(K) G_{0}(K)\left|\left[g_{0}(K)^{-1}+t(K)\right]\right| \\
& \times G_{0}(K) W(K) .
\end{aligned}
$$

The auxiliary transition matrix $t(K)$ itself is obtained by the three-dimensional integral equation

$$
t(K)=w(K)+w(K) g_{0}(K) t(K),
$$

in which the driving term $w(K)$ is derived from the modified four-dimensional interaction $W(K)$ of Eq. (11) according to

$$
w(K):=g_{0}(K)^{-1}\left|G_{0}(K) W(K) G_{0}(K)\right| g_{0}(K)^{-1} .
$$

There is an integral equation for $w(K)$ as there is for $W(K)$, but we do not give it here. We hope that, through our choice (33) for $\widetilde{G}_{0}(K)$, a few terms of the expansion (11), of $W(K)$ in powers of $V(K)$ will dynamically suffice to yield the full result of BSE with satisfactory accuracy. The numerical example of Sec. IV where rapid convergence of $w(K)$ is seen, demonstrates the validity of this expectation.

If the transition matrix $T(K)$ of the BSE has a bound-state pole at total four momentum $K_{B}, \quad K_{B}^{2}=M_{B}^{2}$, the auxiliary three-dimensional transition matrix $t(K)$ also has a boundstate pole at exactly the same $K_{B}$, according to Eq. (34), with the residue $\left|\gamma_{B}\right\rangle$ being the solution of the homogeneous three-dimensional equation

$$
\left|\gamma_{B}\right\rangle=w\left(K_{B}\right) g_{0}\left(K_{B}\right)\left|\gamma_{B}\right\rangle,
$$

corresponding to the inhomogeneous one, Eq. (36). From $\left|\gamma_{B}\right\rangle$, the residue $\left|\Gamma_{B}\right\rangle$ of BSE can be recovered according to Eq. (35)

$$
\left.\left|\Gamma_{B}\right\rangle=W\left(K_{B}\right) G_{0}\left(K_{B}\right)|| \gamma_{B}\right\rangle
$$

as well as the amplitude $\left|\Psi_{B}\right\rangle$ of BSE, i.e.,

$$
\left.\left|\Psi_{B}\right\rangle=G_{0}\left(K_{B}\right) W\left(K_{B}\right) G_{0}\left(K_{B}\right)|| \gamma_{B}\right\rangle,
$$

$$
\begin{aligned}
\left|\Psi_{B}\right\rangle= & {\left[1+\left(G_{0}\left(K_{B}\right)-G_{0}\left(K_{B}\right)\left|g_{0}\left(K_{B}\right)^{-1}\right| G_{0}\left(K_{B}\right)\right) W\left(K_{B}\right)\right] } \\
& \left.\times G_{0}\left(K_{B}\right) \| \gamma_{B}\right\rangle .
\end{aligned}
$$

For the form Eq. (41) of the bound state amplitude, the condition Eq. (38) $\left|\gamma_{B}\right\rangle-w\left(K_{B}\right) g_{0}\left(K_{B}\right)\left|\gamma_{B}\right\rangle=0$ is used. The step from the three-dimensional residue $\left|\gamma_{B}\right\rangle$ to the BetheSalpeter amplitude $\left|\Psi_{B}\right\rangle$ appears predominantly a kinematic one, effected by the operator $G_{0}\left(K_{B}\right) \mid$. Only the second term in Eq. (41) depends on the interaction, and it is expected to be a small correction.

The Bethe-Salpeter amplitude $\left|\Psi_{B}\right\rangle$ is related to the auxiliary three-dimensional $\left|\phi_{B}\right\rangle$, defined by

$$
\left|\phi_{B}\right\rangle:=g_{0}\left(K_{B}\right)\left|\gamma_{B}\right\rangle
$$

and satisfying

$$
\left|\phi_{B}\right\rangle=g_{0}\left(K_{B}\right) w\left(K_{B}\right)\left|\phi_{B}\right\rangle
$$

in an obvious way by

$$
\int d k_{1}^{-}\left\langle k_{1}^{-} \mid \Psi_{B}\right\rangle=\left|\phi_{B}\right\rangle .
$$

The result (44) follows immediately from Eq. (41). The auxiliary bound-state wave function $\left|\phi_{B}\right\rangle$ is the projection of the Bethe-Salpeter amplitude $\left|\Psi_{B}\right\rangle$ to equal light-front individual times $x_{i}^{+}=x^{+}$, taken on the hyperplane $x^{+}=0$.

The Bethe-Salpeter amplitude $\left|\Psi_{B}\right\rangle$ and its threedimensional auxiliary version $\left|\phi_{B}\right\rangle$ still have to be normalized. If the dependence on $K$ of the original interaction $V(K)$ is weak, i.e., $\left[V(K)-V\left(K_{B}\right)\right] /\left(K^{2}-K_{B}^{2}\right) \simeq 0$ and if furthermore the interaction-dependent term in the step from $\left|\phi_{B}\right\rangle$ to $\left|\Psi_{B}\right\rangle$ according to Eq. (41) is small, i.e., $\left|\Psi_{B}\right\rangle$ $\left.\simeq G_{0}\left(K_{B}\right)\left|g_{0}\left(K_{B}\right)^{-1}\right| \phi_{B}\right\rangle$, then

$$
\begin{gathered}
\lim _{K^{2} \rightarrow K_{B}^{2}}\left\langle\Psi_{B}\left|\frac{G_{0}(K)^{-1}-G_{0}\left(K_{B}\right)^{-1}}{K^{2}-K_{B}^{2}}-\frac{V(K)-V\left(K_{B}\right)}{K^{2}-K_{B}^{2}}\right| \Psi_{B}\right\rangle \\
\simeq \lim _{K^{2} \rightarrow K_{B}^{2}}\left\langle\Psi_{B}\left|\frac{G_{0}(K)^{-1}-G_{0}\left(K_{B}\right)^{-1}}{K^{2}-K_{B}^{2}}\right| \Psi_{B}\right\rangle \\
\simeq \lim _{K^{2} \rightarrow K_{B}^{2}}\left\langle\phi_{B}\left|\frac{g_{0}(K)^{-1}-g_{0}\left(K_{B}\right)^{-1}}{K^{2}-K_{B}^{2}}\right| \phi_{B}\right\rangle=1
\end{gathered}
$$

For any further applications, i.e., for predicting physical observables, we now have two equally valid options. We may either work with covariant operators using $\left|\Psi_{B}\right\rangle$ and/or the transition matrix $T(K)$ of the BSE or we may derive effective operators suited for the context of the auxiliary threedimensional bound state $\left|\phi_{B}\right\rangle$ and/or the auxiliary threedimensional transition matrix $t(K)$. We give an example of each of the two possible strategies.

We use the eletroweak current $\mathcal{J}^{\mu}(Q)$ as an example and assume that it connects an initial bound-state Bethe-Salpeter amplitude $\left|\Psi_{B i}\right\rangle$ to a final one $\left|\Psi_{B f}\right\rangle$ in an elastic process. We take $\mathcal{J}^{\mu}(Q)$ to be the current appropriate for the had- 
ronic field theory with four-momentum transfer $Q=K_{B f}$ $-K_{B i}$. The matrix element for describing the process $\left\langle\Psi_{B f}\left|\mathcal{J}^{\mu}(Q)\right| \Psi_{B i}\right\rangle$ can first be derived from the BetheSalpeter amplitude $\left|\Psi_{B}\right\rangle$ of the bound state. Alternatively, it can be obtained from the three-dimensional bound state $\left|\phi_{B}\right\rangle$ by

$$
\left\langle\Psi_{B f}\left|\mathcal{J}^{\mu}\left(K_{B f}-K_{B i}\right)\right| \Psi_{B i}\right\rangle=\left\langle\phi_{B f}\left|j^{\mu}\left(K_{B f}, K_{B i}\right)\right| \phi_{B i}\right\rangle,
$$

with the effective current in three-dimensional space

$$
\begin{aligned}
j^{\mu}\left(K_{f},\right. & \left.K_{i}\right) \\
:= & g_{0}\left(K_{f}\right)^{-1} \mid G_{0}\left(K_{f}\right)\left\{1+W\left(K_{f}\right)\left[G_{0}\left(K_{f}\right)\right.\right. \\
& \left.\left.-G_{0}\left(K_{f}\right)\left|g_{0}\left(K_{f}\right)^{-1}\right| G_{0}\left(K_{f}\right)\right]\right\} \mathcal{J}^{\mu}\left(K_{f}-K_{i}\right) \\
& \times\left\{1+\left[G_{0}\left(K_{i}\right)-G_{0}\left(K_{i}\right)\left|g_{0}\left(K_{i}\right)^{-1}\right| G_{0}\left(K_{i}\right)\right]\right. \\
& \left.\times W\left(K_{i}\right)\right\} G_{0}\left(K_{i}\right) \mid g_{0}\left(K_{i}\right)^{-1} .
\end{aligned}
$$

For the relation between $\left|\Psi_{B}\right\rangle$ and $\left|\phi_{B}\right\rangle$, Eq. (41) is used, which separates the kinematic and dynamic, i.e., interaction dependent, steps in that relation from each other. The bound state has to be calculated for the initial and final four mo- menta $K_{B i}$ and $K_{B f}$. The effective current $j^{\mu}\left(K_{f}, K_{i}\right)$ is predominantly derived kinematically from the covariant one through $g_{0}\left(K_{f}\right)^{-1}\left|G_{0}\left(K_{f}\right) \mathcal{J}^{\mu}\left(K_{f}-K_{i}\right) G_{0}\left(K_{i}\right)\right| g_{0}\left(K_{i}\right)^{-1}$ but it also depends on the interaction $W(K)$ of Eq. (11). If $W(K)$ is not computed in full, but only expanded up to a certain order in the original interaction $V(K)$ of the BSE, the effective current should be expanded consistently up that order.

\section{A NUMERICAL TEST CASE}

We use the bound state of a schematic two-boson system as a test case of the power of the suggested numerical technique. The employed interaction Lagrangian is

$$
\mathcal{L}_{I}=g_{S} \phi_{1}^{\dagger} \phi_{1} \sigma+g_{S} \phi_{2}^{\dagger} \phi_{2} \sigma,
$$

where the bosons with fields $\phi_{1}$ and $\phi_{2}$ have masses $m_{1}$ and $m_{2}$, which we take to be equal, $m_{1}=m_{2}=m$, and the exchanged boson with field $\sigma$ has mass $\mu$. The coupling constant is $g_{S}$.

Using standard techniques in Euclidean space, the homogeneous BSE is solved for the bound-state vertex $\left|\Gamma_{B}\right\rangle$ in the ladder approximation, i.e.,

$$
\left\langle k_{1}^{\prime} \mid \Gamma_{B}\right\rangle=i g_{S}^{2} \int \frac{d^{4} k_{1}}{(2 \pi)^{4}} \frac{\left\langle k_{1} \mid \Gamma_{B}\right\rangle}{\left[\left(k_{1}^{\prime}-k_{1}\right)^{2}-\mu^{2}+i \varepsilon\right]\left(k_{1}^{2}-m^{2}+i \varepsilon\right)\left[\left(K_{B}-k_{1}\right)^{2}-m^{2}+i \varepsilon\right]} .
$$

The solution is calculated in the two-particle c.m. system, i.e., for $K_{B}=\left(M_{B}, \overrightarrow{0}\right)$, and for the ratio of masses $\mu / m$ $=0.5$. Requiring the bound state mass to have a particular value $M_{B}$ fixes the coupling constant $g_{S}$. The fourdimensional bound-state vertex $\left\langle k_{1} \mid \Gamma_{B}\right\rangle$ depends on all Euclidean four components of the momentum $k_{1}$ of boson 1 . The exact four-dimensional bound state amplitude is obtained according to Eq. (10). However, the representation of the vertex and bound-state amplitude in terms of Minkowski momenta is difficult. We do not attempt it.

In contrast, the four-dimensional bound-state amplitude obtained by the numerical technique suggested in Sec. III is available in Minkowski space. We calculate it only approximately by using for the driving term $w\left(K_{B}\right)$ of the auxiliary three-dimensional equation (38), an expansion in orders of the interaction $V(K)$ of BSE in Eqs. (13) and (37), i.e., in powers of the coupling constant $g_{S}$ of the interaction Lagrangian (48). We use the approximation up to the second and fourth powers of $g_{S}$, i.e., $w\left(K_{B}\right) \simeq w^{(2)}\left(K_{B}\right)$ and $w\left(K_{B}\right) \simeq w^{(2)}\left(K_{B}\right)+w^{(4)}\left(K_{B}\right)$. In a time-ordered view, the BSE allows for an exchange of an infinite number of $\sigma$ bosons in stretched configurations. In contrast, the approximative $w^{(2)}\left(K_{B}\right)$ allows only for one exchange [Fig. 1(a)], while $w^{(4)}\left(K_{B}\right)$ allows for two [Fig. 1(b)]. The analytic forms of $w^{(2)}\left(K_{B}\right)$ and $w^{(4)}\left(K_{B}\right)$ are given in Appendixes B and $\mathrm{C}$. The explicit forms of the homogeneous integral equa- tion for $\left|\gamma_{B}\right\rangle$, Eq. (38), for the above approximations in the driving term are given in Appendix D. In order to make a comparision with the exact bound state amplitude we study the following projected forms, i.e.,

$$
\begin{aligned}
f_{\text {exact }}\left(\sqrt{\vec{k}_{1 \perp}^{2}}\right) & :=\int d k_{1}^{-} d k_{1}^{+}\left\langle k_{1} \mid \Psi_{B}\right\rangle \\
& =2 \int d k_{1}^{0} d k_{1}^{3}\left\langle k_{1}^{0} \vec{k}_{1 \perp} k_{1}^{3}\left|G_{0}\left(K_{B}\right)\right| \Gamma_{B}\right\rangle, \\
f_{\text {app }}^{(n)}\left(\sqrt{\vec{k}_{1 \perp}^{2}}\right) & =\int d k_{1}^{+}\left\langle k_{1}^{+} \vec{k}_{1 \perp} \mid \phi_{B}\right\rangle_{\text {app }}^{(n)} \\
& =\int d k_{1}^{+}\left\langle k_{1}^{+} \vec{k}_{1 \perp}\left|g_{0}\left(K_{B}\right)\right| \gamma_{B}\right\rangle_{\text {app }}^{(n)} .
\end{aligned}
$$

The superscripts $(n)$ in Eq. (51) indicate the power of the coupling constant up to which the approximation is carried, i.e., $w\left(K_{B}\right) \simeq \sum_{i=2}^{n} w^{(i)}\left(K_{B}\right)$. The comparision between exact and approximate results is carried out on two levels.

In Fig. 2 the relation between $g_{S}$ and $M_{B}$ is tested for $\mu$ $=0.5 \mathrm{~m}$ against the four-dimensional results. Whereas the exact relation is already satisfactorily reproduced by the approximation based on $w^{(2)}\left(K_{B}\right)$, the approximation based on $w^{(2)}\left(K_{B}\right)+w^{(4)}\left(K_{B}\right)$ improves the agreement. 


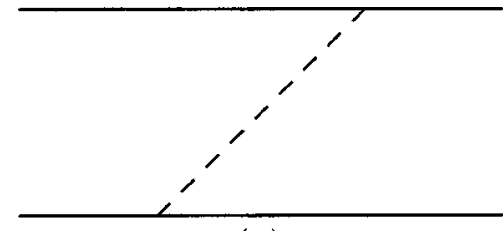

(a)

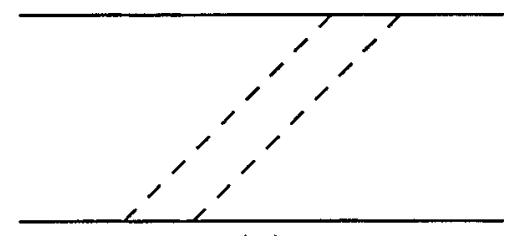

(b)

FIG. 1. Light-front time ordered diagrams for $w^{(2)}(K)$ (a) and $w^{(4)}(K)(\mathrm{b})$, representing the light-front time ordered view of one and two $\sigma$ exchanges, respectively.

In Figs. 3 and 4, the projected bound-state amplitudes $f\left(\sqrt{\vec{k}_{1 \perp}^{2}}\right)$ are compared for two cases. In the first case $M_{B}$ $=0$, i.e., the binding is very strong. It is of the order of the masses of the interacting particles as encountered in quark systems. In the other case $M_{B}=1.98 m$, i.e., the binding is very weak. It is only $2 \%$ of the masses of the interacting particles, as encountered in nuclear systems. In both cases the approximation based on $w^{(2)}\left(K_{B}\right)$ is already quite accurate. The improvement due to the inclusion of $w^{(4)}\left(K_{B}\right)$ is particularly noticeable for the case of strong binding.

The fact that a low-order approximation of $w^{(n)}\left(K_{B}\right)$ works surprisingly well is a virtue of light-front dynamics. It is well known that the analogous approximation scheme in instant-form dynamics has much poorer convergence properties with respect to the number of exchanged $\sigma$ bosons [16].

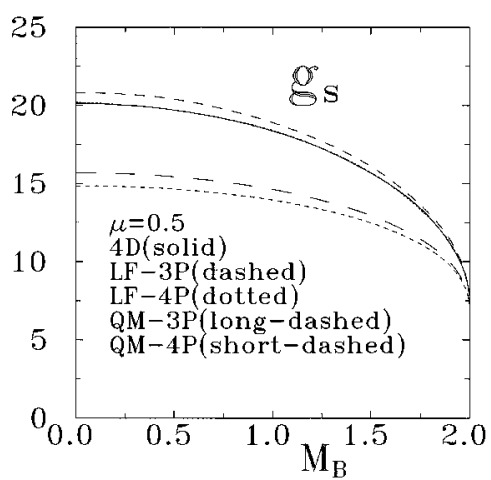

FIG. 2. Results for $g_{S}$ as a function of the two-body bound state mass $M_{B}$ for $\mu=0.5 \mathrm{~m}$. Numerical solution of the covariant fourdimensional BSE (49) (solid curve), the light-front equation (38) with interaction including up to three-particles in the intermediate states, i.e., with $w\left(K_{B}\right) \simeq w^{(2)}\left(K_{B}\right)$ (dashed curve) and including up to four particles in the intermediate states, i.e., with $w\left(K_{B}\right)$ $\simeq w^{(2)}\left(K_{B}\right)+w^{(4)}\left(K_{B}\right)$ (dotted curve). Solution of the quantum mechanics squared mass eigenvalue equation (60), with $w\left(K_{v}\right)$ $\simeq w^{(2)}\left(K_{v}\right)$ (long-dashed curve), and with $w\left(K_{v}\right) \simeq w^{(2)}\left(K_{v}\right)$ $+w^{(4)}\left(K_{v}\right)$ (short-dashed curve) defining the two-particle potential in Eq. (63).
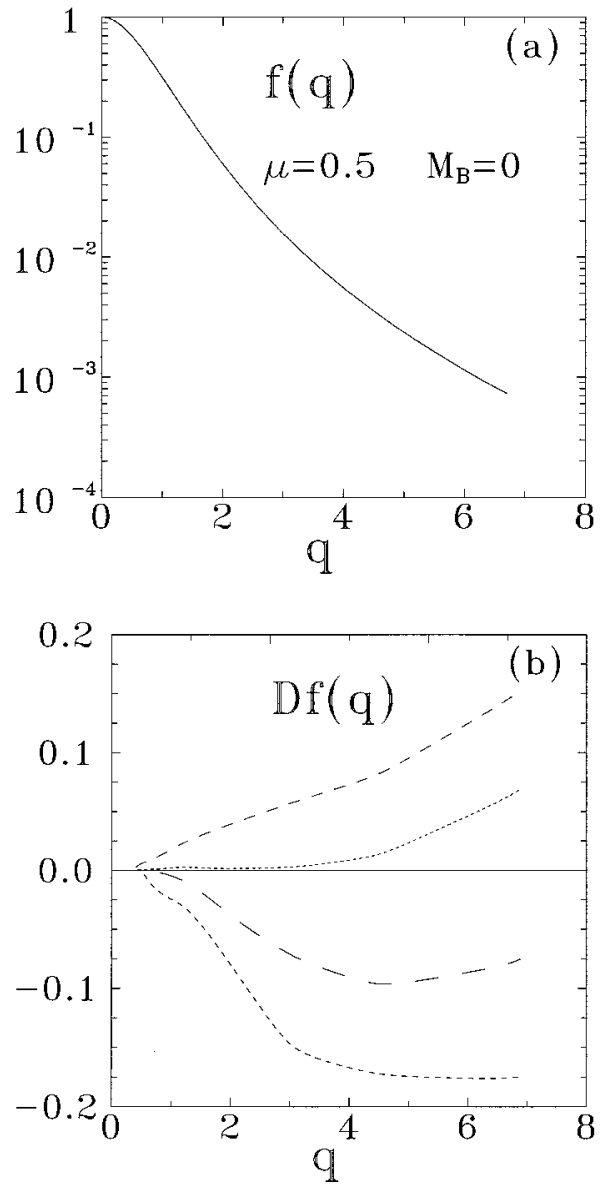

FIG. 3. Results for the transverse momentum distribution $f(q)$ as a function of the transverse component $q$ of the individual fourmomentum, for $M_{B}=0$ and $\mu=0.5 \mathrm{~m}$ : (a) numerical solution of the four-dimensional BSE with $g_{s}=20.14$; (b) relative error of the various approximations with respect to the four-dimensional BSE results, defined by $D f(q)=1-f_{\text {app }}^{(n)}(q) / f_{\text {exact }}(q)$ with $n=2$ and 4 . Results for the light-front equation (38) with an interaction including up to three-particles in the intermediate states, i.e., with $w\left(K_{B}\right)$ $\simeq w^{(2)}\left(K_{B}\right)$ where $g_{s}=20.8$ (dashed curve) and with an interaction including up to four-particles in the intermediate states, i.e., with $w\left(K_{B}\right) \simeq w^{(2)}\left(K_{B}\right)+w^{(4)}\left(K_{B}\right)$ where $g_{s}=20.2$ (dotted curve). Solutions of the quantum mechanics squared mass eigenvalue equation (60), with the two-particle potential in Eq. (63) defined by $w\left(K_{v}\right)$ $\simeq w^{(2)}\left(K_{v}\right)$ where $g_{s}=15.7$ (long-dashed curve), and with $w\left(K_{v}\right)$ $\simeq w^{(2)}\left(K_{v}\right)+w^{(4)}\left(K_{v}\right)$ where $g_{s}=14.9$ (short-dashed curve).

\section{EXTENSION TO FERMIONS}

The Green's function which propagates two fermions disconnectedly contains self-energy corrections as in the case of bosons. They are usually left out of the ladder approximation of interaction. The two-fermion free Green's function then takes the form which we immediately rewrite conveniently as

$$
\begin{gathered}
G_{0}^{F}=\frac{\hat{\boldsymbol{k}}_{1}+m_{1}}{\hat{k}_{1}^{2}-m_{1}^{2}} \frac{\hat{\boldsymbol{k}}_{2}+m_{2}}{\hat{k}_{2}^{2}-m_{2}^{2}}, \\
G_{0}^{F}=\Delta G_{0}^{F}+\left(\hat{\boldsymbol{k}}_{1 o n}+m_{1}\right)\left(\hat{\boldsymbol{k}}_{2 o n}+m_{2}\right) G_{0},
\end{gathered}
$$



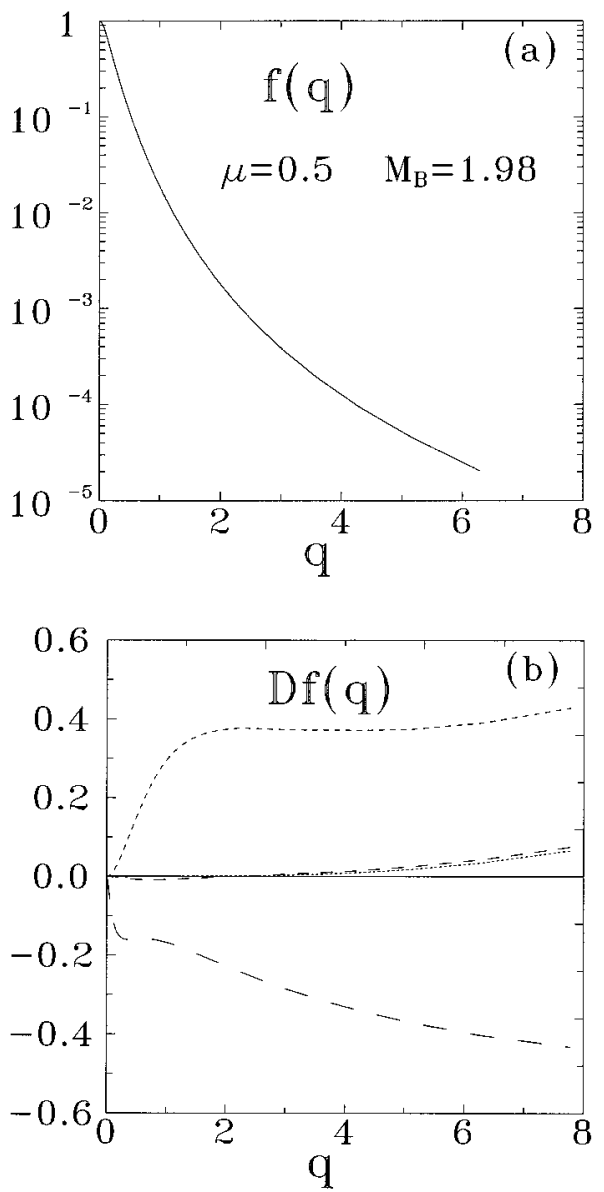

FIG. 4. Results for the transverse momentum distribution $f(q)$ as a function of the transverse component $q$ of the individual fourmomentum, for $M_{B}=1.98 \mathrm{~m}$ and $\mu=0.5 \mathrm{~m}$ : (a) numerical solution of the four-dimensional BSE with $g_{s}=9.03$; (b) relative error of the various approximations in respect to the four-dimensional BSE results, defined by $D f(q)=1-f_{\text {app }}^{(n)}(q) / f_{\text {exact }}(q)$ with $n=2$ and 4 . Results for the light-front equation (38) with interaction including up to three-particles in the intermediate states, i.e., with $w\left(K_{B}\right)$ $\simeq w^{(2)}\left(K_{B}\right)$ where $g_{s}=9.10$ (dashed curve) and with an interaction including up to four-particles in the intermediate states, i.e., with $w\left(K_{B}\right) \simeq w^{(2)}\left(K_{B}\right)+w^{(4)}\left(K_{B}\right)$ where $g_{s}=9.03$ (dotted curve). Solutions of the quantum mechanics squared mass eigenvalue equation (60), with the two-particle potential in Eq. (63) defined by $w\left(K_{v}\right)$ $\simeq w^{(2)}\left(K_{v}\right)$ where $g_{s}=8.33$ (long-dashed curve), and with $w\left(K_{v}\right)$ $\simeq w^{(2)}\left(K_{v}\right)+w^{(4)}\left(K_{v}\right)$ where $g_{s}=8.23$ (short-dashed curve).

where

$$
\hat{k}_{1 o n}^{-}=\frac{\hat{\vec{k}}_{1 \perp}+m_{1}^{2}}{\hat{k}_{1}^{+}} \text {and } \hat{k}_{2 o n}^{-}=\frac{\hat{\vec{k}}_{2 \perp}+m_{2}^{2}}{\hat{k}_{2}^{+}} \text {. }
$$

In Eq. (53) $G_{0}$ is the covariant bosonlike Green's function the paper has worked with in the conceptual development until now. Furthermore, Eq. (53) is the definition of $\Delta G_{0}^{F}$ which contains - except for the particular spin-dependent operators $\left(\hat{\boldsymbol{k}}_{1 \text { on }}+m_{1}\right)$ and $\left(\hat{\boldsymbol{k}}_{2 \text { on }}+m_{2}\right)$ that commute with $G_{0}$-all particular divergences and subtleties connected with the fermion motion. The operator

$$
\Delta G_{0}^{F}=\frac{\gamma_{1}^{+}}{2 k_{1}^{+}} \frac{\hat{k}_{2 o n}+m_{2}}{\hat{k}_{2}^{2}-m_{2}^{2}}+\frac{\hat{k}_{1 o n}+m_{1}}{\hat{k}_{1}^{2}-m_{1}^{2}} \frac{\gamma_{2}^{+}}{2 k_{2}^{+}}+\frac{\gamma_{1}^{+}}{2 k_{1}^{+}} \frac{\gamma_{2}^{+}}{2 k_{2}^{+}}
$$

carries the instantaneous part of the fermion propagators in light-front time. Its is singular under $k_{1}^{-}$integration. We therefore suggest the following strategy for fermions: We apply the reduction to an auxiliary Green's function $\widetilde{G}_{0}$ twice, using the apparatus of Secs. I and II. The operator dependence on the total two-fermion four momentum $K$ is factored out as there. All operators become then parametrically dependent on $K$.

In the first step, the two-fermion Green's function $\left(\hat{\mathbb{k}}_{1 \text { on }}\right.$ $\left.+m_{1}\right)\left(\hat{\boldsymbol{k}}_{2 o n}+m_{2}\right) G_{0}(K)$ is introduced instead of $G_{0}^{F}(K)$. We use formulas (8)-(13) to do this. All the physics of anomalous two-fermion propagation is contained in the new effective interaction $W(K)$ of Eq. (11). Thus, one arrives at a new BSE, corresponding to Eq. (1) after reduction with respect to $K$, with the four-dimensional Green's function $\left(\hat{k}_{1 \text { on }}+m_{1}\right)\left(\hat{k}_{2 \text { on }}+m_{2}\right) G_{0}(K)$ and the new interaction. The resulting two-fermion equation is now solved with the technique as developed for two bosons. This is possible due to the fact that the spin-dependent operator $\left(\hat{\boldsymbol{k}}_{1 \text { on }}+m_{1}\right)\left(\hat{\boldsymbol{k}}_{2 \text { on }}\right.$ $\left.+m_{2}\right)$ also commutes with the auxiliary one $\widetilde{G}_{0}(K)$ $=G_{0}(K)\left|g_{0}(K)^{-1}\right| G_{0}(K)$, i.e.,

$$
\begin{aligned}
& \left(\hat{\boldsymbol{k}}_{1 o n}+m_{1}\right)\left(\hat{\boldsymbol{k}}_{2 o n}+m_{2}\right) \widetilde{G}_{0}(K) \\
& \quad=\widetilde{G}_{0}(K)\left(\hat{\boldsymbol{k}}_{1 o n}+m_{1}\right)\left(\hat{\boldsymbol{k}}_{2 o n}+m_{2}\right) .
\end{aligned}
$$

This idea will not be further developed in this paper, but indicates that the scope of the method extends beyond the two-boson system.

\section{RELATION TO LIGHT-FRONT QUANTUM MECHANICS}

Sections I-IV used the notion of a bound state, but scattering states were not introduced. The latter could have been introduced in the BSE (1) as well as in the auxiliary threedimensional equation (36) for $t(K)$ with the global propagator $g_{0}(K)$. Given an initial two-particle plane-wave state $\left|k_{1}^{+} \vec{k}_{1 \perp} K_{o n}\right\rangle$ with total momentum $K_{\text {on }}$ and light-front "energy',

$$
K_{o n}^{-}=\frac{\vec{k}_{1 \perp}^{2}+m_{1}^{2}}{k_{1}^{+}}+\frac{\left(\vec{K}_{\perp}-\vec{k}_{1 \perp}\right)^{2}+m_{2}^{2}}{K^{+}-k_{1}^{+}},
$$

one may define the corresponding three-dimensional scattering state $\left|\phi^{(+)}\left(k_{1}^{+} \vec{k}_{1 \perp} K_{o n}\right)\right\rangle$ with outgoing light-front boundary conditions as the solution of a standard LippmanSchwinger type of equation, i.e.,

$$
\begin{aligned}
\left|\phi^{(+)}\left(k_{1}^{+} \vec{k}_{1 \perp} K_{o n}\right)\right\rangle= & \left|k_{1}^{+} \vec{k}_{1 \perp} K_{o n}\right\rangle+g_{0}\left(K_{o n}\right) w\left(K_{o n}\right) \\
& \times\left|\phi^{(+)}\left(k_{1}^{+} \vec{k}_{1 \perp} K_{o n}\right)\right\rangle
\end{aligned}
$$


with four-momentum $K_{o n}=\left(K_{o n}^{-}, K^{+}, \vec{K}_{\perp}\right)$. The relation to the auxiliary transition operator $t(K)$ is obvious,

$$
t\left(K_{\text {on }}\right)\left|k_{1}^{+} \vec{k}_{1 \perp} K_{\text {on }}\right\rangle=w\left(K_{\text {on }}\right)\left|\phi^{(+)}\left(k_{1}^{+} \vec{k}_{1 \perp} K_{\text {on }}\right)\right\rangle .
$$

Furthermore, it satisfies the homogeneous equation

$$
\left[g_{0}\left(K_{o n}\right)^{-1}-w\left(K_{o n}\right)\right]\left|\phi^{(+)}\left(k_{1}^{+} \vec{k}_{1 \perp} K_{o n}\right)\right\rangle=0
$$

in the same way as the auxiliary bound state $\left|\phi_{B}\right\rangle$ of Eq. (38) does, i.e.,

$$
\left[g_{0}\left(K_{B}\right)^{-1}-w\left(K_{B}\right)\right]\left|\phi_{B}\right\rangle=0 .
$$

Equations (58) and (59) formally look similar to the eigenvalue equations of quantum mechanics with the only difference being that the two-particle interaction $w(K)$ depends on the eigenvalue. Untill now the relationship to quantum mechanics has indeed been entirely formal. The states $\left|\phi_{B}\right\rangle$ and $\left|\phi^{(+)}\left(k_{1}^{+} \vec{k}_{1 \perp} K_{o n}\right)\right\rangle$ and the corresponding transition matrix have significance only as quantities from which the solutions of the BSE can be obtained with comparative ease. On the other hand, at this stage a quantum-mechanical description of the two-particle system can be given which corresponds dynamically to the underlying field-theoretic one, though it is by no means equivalent to it.

Quantum-mechanical two-particle states $|\varphi\rangle$ are required to satisfy the eigenvalue equation for the squared mass operator

$$
\left[M_{0}^{2}+v\left(K^{+}, \vec{K}_{\perp}\right)\right]|\varphi\rangle=M_{B}^{2}|\varphi\rangle
$$

where the squared free-mass operator is

$$
M_{0}^{2}=\frac{\hat{\vec{k}}_{1 \perp}^{2}+m_{1}^{2}}{\hat{x}}+\frac{\left(\vec{K}_{\perp}-\hat{\vec{k}}_{1 \perp}\right)^{2}+m_{2}^{2}}{1-\hat{x}} .
$$

and $\hat{x}=\hat{k}_{1}^{+} / \hat{K}^{+}$. The states are elements of a Hilbert-space spanned by the free-particle on-mass-shell basis states. Boundary conditions must be imposed on the solutions of Eq. (60) in order to make them acceptable. Bound-state and scattering state solutions to the mass squared operator equation exist and are orthonormalized. The orthonormalization for scattering states is of the $\delta$-function type. The states have a probability interpretation. The quantum mechanical boundstate normalization is

$$
\left\langle\varphi_{B} \mid \varphi_{B}\right\rangle=1
$$

The two-particle potential $v\left(K^{+}, \vec{K}_{\perp}\right)$ is independent of the eigenvalue $K^{-}$, the eigenvalue $K_{B}^{-}$to be calculated for the bound state and the eigenvalue $K_{\text {on }}^{-}$prescribed for the scattering states; the potential is Hermitian- it is instantaneos in light-front time; it conserves the kinematic components $\left(K^{+}, \vec{K}_{\perp}\right)$ of the total two-particle four-momentum $K$. In quantum mechanics $v\left(K^{+}, \vec{K}_{\perp}\right)$ may be parametrized by fitting it to observables. If contact is attempted to a corresponding field theory a standard form of identification is

$$
\begin{aligned}
\left\langle k_{1}^{\prime+} \vec{k}_{1 \perp}^{\prime}\left|v\left(K^{+}, \vec{K}_{\perp}\right)\right| k_{1}^{+} \vec{k}_{1 \perp}\right\rangle & =i \sqrt{\frac{K^{+}}{k_{1}^{\prime+}\left(K^{+}-k_{1}^{\prime+}\right)}} \\
& \times\left\langle k_{1}^{\prime+} \vec{k}_{1 \perp}^{\prime}\left|w\left(K_{v}\right)\right| k_{1}^{+} \vec{k}_{1}\right\rangle \sqrt{\frac{K^{+}}{k_{1}^{+}\left(K^{+}-k_{1}^{+}\right)}}
\end{aligned}
$$

with

$$
K_{v}=\left(\frac{1}{2} K_{o n}^{\prime-}+\frac{1}{2} K_{o n}^{-}, K^{+}, \vec{K}_{\perp}\right) .
$$

The relativistic quantum-mechanical potential $v\left(K^{+}, \vec{K}_{\perp}\right)$ is defined in the framework of light-front dynamics. The value $K_{o n}^{-}$is defined in the context of Eq. (60). This choice guarantees that the $S$ matrix calculated field theoretically to first order in $w(K)$ and calculated quantum mechanically to first order in $v\left(K^{+}, \vec{K}_{\perp}\right)$ are identical. The $S$ matrix carries a $\delta$ function for light-front energy $K^{-}$between initial and final states. The definition of Eq. (63) removes that $\delta$ function from $v\left(K^{+}, \vec{K}_{\perp}\right)$ and allows for general off $K^{-}$-shell matrix elements. Thus, Eq. (64) implies a very particular off-shell extension. This procedure of identification-it is no derivation - is standard for the instant-form of quantum mechanics, e.g., when the one-boson exchange potential between nucleons is introduced. This paper extends that procedure to light-front quantum mechanics. Furthermore, the potential is usually defined in the two-particle c.m. system, i.e., for $\vec{K}_{\perp}=0$, and is considered unchanged in moving systems, i.e., independent of $\vec{K}_{\perp}$ and $K^{+}$.

The identification (63) motivates a quantum-mechanical potential. It does not attempt to derive it. The goal of the identification is not to simulate exact solutions of the BSE but to be in a best accord with a chosen physics criterion. A quantum mechanics description has different objectives than matching a field-theory result. It rather attempts to describe many-particle systems with the same rules once it has done so satisfactorily for the two-particle system with the same rules. Thus, when the quantum-mechanical potential cannot be derived completely, as is the case in hadronic physics, the potential is tuned to known experimental properties of the two-particle system and then considered a vehicle which carries that two-particle information to many-particle systems. Despite the particular many-particle aspect of quantum mechanics, a study of its predictive quality even for the twoparticle system is interesting. Figures 2-4 perform such study for the two-boson system of Sec. IV. The bound state constitutes an especially stringent test. For the instantaneous choice, the approximation, $K^{-}=K_{o n}^{-}>m_{1}+m_{2}$ in the interaction in the c.m. system is quite severe, because in this case field theory requires $K^{-}=K_{B}^{-}<m_{1}+m_{2}$. The relation between the coupling constant $g_{S}$ and the bound-state mass $M_{B}$ and the dependence of the bound-state wave function $f(q)$ on the momentum $q=\sqrt{\vec{k}_{\perp}^{2}}$ are compared in the field theoretic and quantum-mechanical descriptions. Results are stud- 
ied for the approximations $w(K) \simeq w^{(2)}(K)$ and $w(K)$ $\simeq w^{(2)}(K)+w^{(4)}(K)$ up to second order and fourth-order in the coupling constant $g_{S}$. The quantum-mechanical binding energy and wave function preserve most field-theoretic characteristics, expectedly better in the case of small binding rather than in the case of strong binding. The quantummechanical choice of the potential is usually based on the one-boson exchange, i.e., on the approximation $w(K)$ $\simeq w^{(2)}(K)$. We are happy to find that this identification accounts better for the field-theoretic results than the choice based on $w(K) \simeq w^{(2)}(K)+w^{(4)}(K)$.

Instead of solving Eq. (60), its formal identity with the energy eigenvalue problem for a nonrelativistic Hamiltonian is often exploited [17] and $\left|\varphi_{B}\right\rangle$ is applied directly in the framework of light-front quantum mechanics.

The response of the quantum-mechanical system to an electromagnetic probe is given by a four-vector current $j_{v}^{\mu}\left(K^{\prime+}-K^{+}, \vec{K}_{\perp}^{\prime}-\vec{K}_{\perp}\right)$ which, as the quantum-mechanical potential is a three-dimensional operator and it depends on the three-dimensional momentum transfer $\left(Q^{+}, \vec{Q}_{\perp}\right)=\left(K^{\prime+}\right.$ $\left.-K^{+}, \vec{K}_{\perp}^{\prime}-\vec{K}_{\perp}\right)$. As in the case of the potential, contact can be attempted with the corresponding field theory. A possible identification is

$$
\begin{aligned}
\left\langle k_{1}^{\prime+} \vec{k}_{1 \perp}^{\prime}\left|j_{v}^{\mu}\left(K^{\prime+}-K^{+}, \vec{K}_{\perp}^{\prime}-\vec{K}_{\perp}\right)\right| k_{1}^{+} \vec{k}_{1 \perp}\right\rangle & \\
:= & \sqrt{\frac{K^{+}}{k_{1}^{\prime+}\left(K^{+}-k_{1}^{\prime+}\right)}}\left\langle k_{1}^{\prime+} \vec{k}_{1 \perp}^{\prime}\left|j^{\mu}\left(K_{v}^{\prime}-K_{v}\right)\right| k_{1}^{+} \vec{k}_{1 \perp}\right\rangle \\
& \times \sqrt{\frac{K^{+}}{k_{1}^{+}\left(K^{+}-k_{1}^{+}\right)}}
\end{aligned}
$$

with

$$
\begin{gathered}
K_{v}=\left(K_{o n}, K^{+}, \vec{K}_{\perp}\right), \\
K_{v}^{\prime}=\left(K_{o n}^{\prime}, K^{\prime+}, \vec{K}_{\perp}^{\prime}\right) .
\end{gathered}
$$

The field-theoretic $j^{\mu}\left(K^{\prime}-K\right)$ is the one of Eq. (47) in Sec. III. It contains the field-theoretic interaction in the form of $w(K)$. The quantum-mechanical current $j_{v}^{\mu}\left(K^{\prime+}-K^{+}, \vec{K}_{\perp}^{\prime}\right.$ $\left.-\vec{K}_{\perp}\right)$, is derived in the special case of elastic scattering between bound states. Thus, the identification of Eq. (65) is not consistent with the choice of Eq. (63), which guaranteed the agreement of the field-theoretic and the quantummechanical $S$ matrix in first order in the interaction. Nevertheless, the quantum-mechanical current $j_{v}^{\mu}\left(K^{\prime+}-K^{+}, \vec{K}_{\perp}^{\prime}\right.$ $\left.-\vec{K}_{\perp}\right)$ can be meaningfully studied and separated into interaction-free single particle and interaction-dependent two-particle pieces. Thus, the definition of Eq. (65) implicitly contains a possible quantum-mechanical definition of an interaction-dependent two-particle current. At this stage, the standard definition based on the identification of the $S$ matrix could also be given [18]. It also exploits the formal identity of the eigenvalue problem with a nonrelativistic Hamiltonian with equations similar to Eq. (60), but it then identifies the nonrelativistic bound state with the solution $\left|\phi_{B}\right\rangle$ of Eq. (59), the auxiliary field-theoretic bound state for the BSE. Thus, the calculation of the electromagnetic deuteron form factors in Ref. [18] is performed in the field-theoretic spirit of Eq. (59). The two-particle current operators of pion range in Ref. [18] should not be confused with the quantum-mechanical interaction-dependent two-particle currents of this section.

\section{CONCLUSION}

The paper suggests a calculational procedure for solving the BSE with comparative ease and in principle, with any desired accuracy. The procedure is based on an auxiliary three-dimensional integral equation, in the framework of light-front dynamics, whose solution then yields the result of the BSE by quadrature. The intermediate auxiliary quantities do not display covariance; covariance is restored in the final step to the full result of BSE.

The calculational procedure is exact, but it also offers an efficient approximative scheme: Only particles propagate. Antiparticles do not. Antiparticle propagation is relegated to the effective interaction. The convergence with repect to the number of exchanged particles mediating the interaction appears to be rapid. Though only an indication of that fact comes from the simple test case of a BSE bound state in ladder approximation, it is supported by the similar result of Ref. [16] for the corresponding scattering amplitude. Calculational improvements are possible in a systematic manner. Thus, as a further and physically more interesting consequence, the solution of the BSE for bound state and scattering up to fourth order in the coupling constant, i.e., in ladder and crossed ladder approximation and with the inclusion of self-energy corrections is obtained based on a simplifying three-dimensional calculational procedure. The procedure capitalizes on beneficial properties of light-front dynamics. It should be an interesting alternative to the Gross approach [6] which is also three dimensional and which has been suggested to include the cross-ladder exchanges approximately.

The calculational procedure is general, though it is given in this paper for an interacting two-boson system only. The ideas needed for an extension to fermions are developed but important technical details have not yet been worked out and unforeseen difficulties may still arise. The problem of rotational invariance in light-front dynamics will become especially acute for fermions when spin and orbital angular momentum are to be coupled. The auxiliary three-dimensional quantities will then be hampered by their lack of rotational invariance. We strongly believe, however, that the final step to the covariant result of BSE will overcome that difficulty.

The auxiliary three-dimensional quantities, i.e., the operators and equations, that mediate the solution of the BSE, are close in spirit to relativistic quantum mechanics. The paper also discusses this relation. First, only particles, and not antiparticles, propagate in the three-dimensional equations and in quantum mechanics. Second, the quantum-mechanical interaction is an instantaneous potential, the corresponding interaction $w(K)$ in the three-dimensional equation is not. However, this paper finds that the instantaneous choice for the potential does not distort the physics of the underlying 
field theory. Thus, the relation between quantum mechanics and field theory can be made close. However, compared to field theory, quantum mechanics has the virtue of an instant extension to many-particle systems: Barring many-particle forces, the quantum-mechanical interaction is additive in the instantaneous pairwise potentials. In fact, the conceptual strategy of quantum mechanics often is to tune away shortcomings of the chosen instantaneous potential by adjusting undetermined phenomenological parameters to vital known experimental properties of the considered two-particle system. In this way the potential carries the accepted knowledge on the two-particle system over to many-particle systems.

The paper left open the relationship of the theoretical apparatus developed to realistic physics problems. We have in mind applications to hadronic and subhadronic systems. The concept of light-front wave functions was applied in the context of nuclear physics to describe the deuteron [17] and the discussion of its properties in the light front continues to the present [19]. The BSE is supposed to yield the bound states and the scattering amplitudes of those two-particle systems. In contrast, the response of such a two-particle system towards an eletroweak probe is considered in perturbation theory. The required matrix element is determined by the field-theoretic current between states of the BSE. The paper offers two equivalent routes for calculation: Either the covariant states of BSE are constructed and then used in their four-dimensional form together with the field-theoretic current or that field-theoretic current is reduced to an auxiliary three-dimensional one and used with the auxiliary threedimensional states. Both calculational schemes are equivalent field-theoretic ones. However, the latter calculational scheme is close in spirit to the quantum-mechanical one. Quantum mechanics requires the definition of two-particle exchange currents; its definition is also sketched.

\section{ACKNOWLEDGMENTS}

The authors acknowledge the partial support from the CAPES/DAAD/Probral Project No. 015/95. Part of this work was done during the visits of T.F. and P.U.S. They thank the hospitality at the respective host institutions. Furthermore, the work was supported by a Brazilian graduate-student grant (J.H.O.S.) from FAPESP and by research grants (B.V.C. and T.F.) from CNPq and FAPESP.

\section{APPENDIX A: EVALUATION OF AUXILIARY QUANTITIES}

The operators $G_{0}(K) \mid g_{0}(K)^{-1}$ and $g_{0}^{-1} \mid G_{0}(K)$ connect three-dimensional and four-dimensional basis states. The two operators are related by conjugation; we therefore discuss only one, i.e., $G_{0}(K) \mid g_{0}(K)^{-1}$.

The momentum space matrix elements of $G_{0}(K) \mid g_{0}(K)^{-1}$ for $K^{+}>0$, are

$$
\begin{aligned}
\left\langle k_{1}^{\prime-} k_{1}^{\prime+} \vec{k}_{1 \perp}^{\prime}\left|G_{0}(K)\right| g_{0}(K)^{-1} \mid k_{1}^{+} \vec{k}_{1 \perp}\right\rangle & =\frac{i}{2 \pi} \frac{\delta\left(k_{1}^{\prime+}-k_{1}^{+}\right) \delta\left(\vec{k}_{1 \perp}^{\prime}-\vec{k}_{1 \perp}\right)}{\left(k_{1}^{\prime-}-\frac{\vec{k}_{1 \perp}^{\prime 2}+m_{1}^{2}-i o}{k_{1}^{\prime+}}\right)} \\
& \times \frac{\left(K^{-}-k_{1 o n}^{-}-k_{2 o n}^{-}+i o\right) \theta\left(K^{+}-k_{1}^{+}\right) \theta\left(k_{1}^{+}\right)}{\left(K^{-}-k_{1}^{\prime-}-\frac{\left(\vec{K}_{\perp}-\vec{k}_{1 \perp}^{\prime}\right)^{2}+m_{2}^{2}-i o}{K^{+}-k_{1}^{\prime+}}\right)} .
\end{aligned}
$$

When the avaliable light-front "energy" $K^{-}$is not on shell, i.e., $K^{-} \neq k_{1 \text { on }}^{-}+k_{2 \text { on }}^{-}$, the evaluation of the matrix element in Eq. (A1) is standard. The two singular propagators

$$
\begin{gathered}
\left(k_{1}^{\prime-}-\frac{\vec{k}_{1 \perp}^{\prime 2}+m_{1}^{2}-i o}{k_{1}^{\prime+}}\right)^{-1} \text { and } \\
\left(K^{-}-k_{1}^{\prime-}-\frac{\left(\vec{K}_{\perp}-\vec{k}_{1 \perp}^{\prime+}\right)^{2}+m_{2}^{2}-i o}{K^{+}-k_{1}^{\prime+}}\right)^{-1}
\end{gathered}
$$

can be rewritten as a $\delta$ function and principal-part singularity; integration on $k_{1}^{\prime-}$ can be carried out with usual techniques.

A problem arises, when the avaliable light-front "energy" $K^{-}$is on-shell, i.e., $K^{-}=K_{o n}^{-}=k_{1 o n}^{-}+k_{2 o n}^{-}$. Without losing generality, we will have to suppose that $K^{+}>0$ and $k_{1}^{+}>0$. Then, $K^{-}-k_{1 o n}^{-}-k_{2 o n}^{-}+i o=+i o$ and the limiting process of going to the real axis must be performed with care. However, in this situation the matrix element will always be integrated with respect to $k_{1}^{\prime-}$, over a function $f\left(k_{1}^{\prime-}\right)$ still to be determined and, unfortunately with unknown analyticity properties, i.e.,

$$
\begin{aligned}
\int d k_{1}^{\prime-} f\left(k_{1}^{\prime-}\right)\left\langle k_{1}^{\prime-} k_{1}^{\prime+} \vec{k}_{1 \perp}^{\prime}\left|G_{0}(K)\right| g_{0}(K)^{-1} \mid k_{1}^{+} \vec{k}_{1 \perp}\right\rangle \\
=\frac{i}{2 \pi} \delta\left(k_{1}^{\prime+}-k_{1}^{+}\right) \delta\left(\vec{k}_{1 \perp}^{\prime}-\vec{k}_{1 \perp}\right) \int d k_{1}^{\prime-} \\
\quad \times \frac{f\left(k_{1}^{\prime-}\right)}{\left(k_{1}^{\prime-}-k_{1 o n}^{-}+i o\right)} \frac{1}{\left(K^{-}-k_{1}^{\prime-}-k_{2 o n}^{-}+i o\right)} \\
\quad \times\left(K^{-}-k_{1 o n}^{-}-k_{2 o n}^{-}+i o\right) .
\end{aligned}
$$

Without any loss of generality, we can think of $f\left(k_{1}^{\prime-}\right)$ as being split into a part $f_{u h p}\left(k_{1}^{\prime-}\right)$ having singularities only in the upper half $k_{1}^{\prime-}$ plane and a part $f_{l h p}\left(k_{1}^{\prime-}\right)$ having singularities only in the lower half $k_{1}^{\prime-}$ plane, i.e.,

$$
f\left(k_{1}^{\prime-}\right)=f_{u h p}\left(k_{1}^{\prime-}\right)+f_{l h p}\left(k_{1}^{\prime-}\right) .
$$

In the case that there is a part with poles simultaneously in both half planes, they can be fully separated, i.e., 


$$
\begin{aligned}
g\left(k_{1}^{\prime-}\right) & \frac{1}{k_{1}^{\prime-}-\alpha_{1}-i \alpha_{2}} \frac{1}{k_{1}^{\prime-}-\beta_{1}+i \beta_{2}} \\
= & g\left(k_{1}^{\prime-}\right) \frac{1}{(\alpha-\beta)+i\left(\alpha_{2}+\beta_{2}\right)} \\
& \times\left[\frac{1}{k_{1}^{\prime-}-\alpha_{1}-i \alpha_{2}}-\frac{1}{k_{1}^{\prime-}-\beta_{1}+i \beta_{2}}\right]
\end{aligned}
$$

with $g\left(k_{1}^{\prime-}\right)$ being singularity free. The integration in Eq. (A2) can now be carried out using Cauchy's theorem:

$$
\begin{aligned}
\int d k_{1}^{\prime-} f\left(k_{1}^{\prime-}\right)\left\langle k_{1}^{\prime-} k_{1}^{\prime+} \vec{k}_{1 \perp}^{\prime}\left|G_{0}(K)\right| g_{0}(K)^{-1} \mid k_{1}^{+} \vec{k}_{1 \perp}\right\rangle \\
=\delta\left(k_{1}^{\prime+}-k_{1}^{+}\right) \delta\left(\vec{k}_{1 \perp}^{\prime}-\vec{k}_{1 \perp}\right)\left(K^{-}-k_{1 o n}^{-}-k_{2 o n}^{-}+i o\right) \\
\quad \times\left[f_{u h p}\left(k_{1 o n}^{-}\right) \frac{1}{K^{-}-k_{1 o n}^{-}-k_{2 o n}^{-}+i o}\right. \\
\left.+f_{l h p}\left(K^{-}-k_{2 o n}^{-}\right) \frac{1}{K^{-}-k_{2 o n}^{-}-k_{1 o n}^{-}+i o}\right]
\end{aligned}
$$

$$
\begin{aligned}
= & \delta\left(k_{1}^{\prime+}-k_{1}^{+}\right) \delta\left(\vec{k}_{1 \perp}^{\prime}-\vec{k}_{1 \perp}\right)\left[f_{u h p}\left(k_{1 o n}^{-}\right)\right. \\
& \left.+f_{\text {lhp }}\left(K^{-}-k_{2 o n}^{-}\right)\right] .
\end{aligned}
$$

We note that propagators cancel and no singularity remains. However, the result (A5) is for practical purposes useless, since the split into two parts with disjoint singularities is not known in a numerical calculation. If, however, the light-front "energy" is on-shell, $K^{-}=K_{o n}^{-}$, then the two terms can be recombined to the original function, i.e.,

$$
\begin{aligned}
& \qquad \begin{array}{l}
\int k_{1}^{\prime-} f\left(k_{1}^{\prime-}\right)\left\langle k_{1}^{\prime-} k_{1}^{\prime+} \vec{k}_{1 \perp}^{\prime}\left|G_{0}(K)\right| g_{0}(K)^{-1} \mid k_{1}^{+} \vec{k}_{1 \perp}\right\rangle \\
=\delta\left(k_{1}^{\prime+}-k_{1}^{+}\right) \delta\left(\vec{k}_{1 \perp}^{\prime}-\vec{k}_{1 \perp}\right) f\left(k_{1 o n}^{-}\right)
\end{array} \\
& \text {for } K^{-}=K_{o n}^{-} \text {. }
\end{aligned}
$$

\section{APPENDIX B: INTERACTION IN FIRST ORDER}

The interaction $w(k)$, defined by Eqs. (37) and (11) to lowest order of the driving term $V(K)$, is given by

$$
w^{(2)}(K)=g_{0}(K)^{-1}\left|G_{0}(K) V(K) G_{0}(K)\right| g_{0}(K)^{-1},
$$

where the matrix element of the operator $\left|G_{0}(K) V(K) G_{0}(K)\right|$ is

$$
\begin{aligned}
\left\langle k_{1}^{\prime+} \vec{k}_{1 \perp}^{\prime}\left\|G_{0}(K) V(K) G_{0}(K)\right\| k_{1}^{+} \vec{k}_{1 \perp}\right\rangle & =i \frac{\left(i g_{S}\right)^{2}}{(2 \pi)^{2}} \int d k_{1}^{\prime-} d k_{1}^{-} \frac{1}{k_{1}^{\prime+}\left(K^{+}-k_{1}^{\prime+}\right)} \frac{1}{\left.k_{1}^{\prime-}-\frac{\vec{k}_{1 \perp}^{\prime 2}+m_{1}^{2}-i o}{k_{1}^{\prime+}}\right)} \frac{1}{\left(K^{-}-k_{1}^{\prime-}-\frac{\left(\vec{K}_{\perp}-\vec{k}_{1 \perp}^{\prime 2}\right)+m_{2}^{2}-i o}{K^{+}-k_{1}^{\prime+}}\right)} \\
& \times \frac{1}{\left(k_{1}^{\prime+}-k_{1}^{+}\right)} \frac{1}{\left(k_{1}^{\prime-}-k_{1}^{-}-\frac{\left(\vec{k}_{1}^{\prime}-\vec{k}_{1 \perp}\right)^{2}+\mu^{2}-i o}{k_{1}^{\prime+}-k_{1}^{+}}\right)} \frac{1}{k_{1}^{+}\left(K^{+}-k_{1}^{+}\right)} \frac{1}{\left(k_{1}^{-}-\frac{\vec{k}_{1 \perp}^{2}+m_{1}^{2}-i o}{k_{1}^{+}}\right)} \\
& \times \frac{1}{\left(K^{-}-k_{1}^{-}-\frac{\left(\vec{K}_{\perp}-\vec{k}_{1 \perp}\right)^{2}+m_{2}^{2}-i o}{K^{+}-k_{1}^{+}}\right)} .
\end{aligned}
$$

The double integration in $k^{-}$in Eq. (B2) is performed analytically using Cauchy's theorem and the condition $K^{+}>0$. The integration is nonzero for $K^{+}>k_{1}^{\prime+}>0$ and $K^{+}>k_{1}^{+}>0$. Two possibilities also appear for $\sigma$ forward propagation. For $k_{1}^{+}$ $>k_{1}^{\prime}$, a $\sigma$ is emitted by particle 1 and otherwise absorbed:

$$
\begin{aligned}
\left\langle k_{1}^{\prime+} \vec{k}_{1 \perp}^{\prime} \|\right. & \left.G_{0}(K) V(K) G_{0}(K) \| k_{1}^{+} \vec{k}_{1 \perp}\right\rangle \\
= & \left(i g_{S}\right)^{2} \frac{i \theta\left(K^{+}-k_{1}^{\prime+}\right) \theta\left(k_{1}^{\prime+}\right)}{k_{1}^{\prime+}\left(K^{+}-k_{1}^{\prime+}\right)\left(K^{-}-k_{1 o n}^{\prime-}-k_{2 o n}^{\prime-}+i o\right)} \\
& \times\left(\frac{\theta\left(k_{1}^{+}-k_{1}^{\prime+}\right)}{\left(k_{1}^{+}-k_{1}^{\prime+}\right)} \frac{i}{\left(K^{-}-k_{1 o n}^{\prime-}-k_{2 o n}^{-}-k_{\sigma o n}^{\prime-}+i o\right)}+\frac{\theta\left(k_{1}^{\prime+}-k_{1}^{+}\right)}{\left(k_{1}^{\prime+}-k_{1}^{+}\right)} \frac{i}{\left(K^{-}-k_{1 o n}^{-}-k_{2 o n}^{\prime}-k_{\sigma o n}^{-}+i o\right)}\right) \\
& \times \frac{i \theta\left(K^{+}-k_{1}^{+}\right) \theta\left(k_{1}^{+}\right)}{k_{1}^{+}\left(K^{+}-k_{1}^{+}\right)\left(K^{-}-k_{1 o n}^{-}-k_{2 o n}^{-}+i o\right)},
\end{aligned}
$$


where the light-front "energies" of the intermediate states of the individual particles are given by

$$
\begin{gathered}
k_{1 o n}^{\prime-}=\frac{\vec{k}_{1 \perp}^{\prime 2}+m_{1}^{2}}{k_{1}^{\prime+}}, \\
k_{1 o n}^{-}=\frac{\vec{k}_{1 \perp}^{2}+m_{1}^{2}}{k_{1}^{+}}, \\
k_{2 o n}^{\prime-}=\frac{\left(\vec{K}_{\perp}-\vec{k}_{1 \perp}^{\prime}\right)^{2}+m_{2}^{2}}{K^{+}-k_{1}^{\prime+}}, \\
k_{2 o n}^{-}=\frac{\left(\vec{K}_{\perp}-\vec{k}_{1 \perp}\right)^{2}+m_{2}^{2}}{K^{+}-k_{1}^{+}},
\end{gathered}
$$

$$
\begin{aligned}
& k_{\sigma o n}^{\prime-}=\frac{\left(\vec{k}_{1 \perp}^{\prime}-\vec{k}_{1 \perp}\right)^{2}+\mu^{2}}{k_{1}^{+}-k_{1}^{\prime+}}, \\
& k_{\sigma o n}^{-}=\frac{\left(\vec{k}_{1 \perp}^{\prime}-\vec{k}_{1 \perp}\right)^{2}+\mu^{2}}{k_{1}^{\prime+}-k_{1}^{+}} .
\end{aligned}
$$

The global three-particle propagator for 1,2, and $\sigma$ appears in Eq. (B3), in two cases: when $\sigma$ is either emitted or absorbed by particle 1 .

The matrix element $\left\langle k_{1}^{\prime+} \vec{k}_{1 \perp}^{\prime}\left|w^{(2)}(K)\right| k_{1}^{+} \vec{k}_{1 \perp}\right\rangle$ is obtained from Eq. (B3) by multiplying both sides by the matrix element of the operator $g_{0}(K)^{-1}$ from Eq. (25):

$$
\begin{aligned}
\left\langle k_{1}^{\prime+} \vec{k}_{1 \perp}^{\prime}\left|w^{(2)}(K)\right| k_{1}^{+} \vec{k}_{1 \perp}\right\rangle= & \left(i g_{S}\right)^{2} \frac{\theta\left(k_{1}^{+}-k_{1}^{\prime+}\right)}{\left(k_{1}^{+}-k_{1}^{\prime+}\right)} \frac{i}{\left(K^{-}-k_{1 o n}^{\prime-}-k_{2 o n}^{-}-k_{\sigma o n}^{\prime-}+i o\right)} \\
& +\left(i g_{S}\right)^{2} \frac{\theta\left(k_{1}^{\prime+}-k_{1}^{+}\right)}{\left(k_{1}^{\prime+}-k_{1}^{+}\right)} \frac{i}{\left(K^{-}-k_{1 o n}^{-}-k_{2 o n}^{\prime-}-k_{\sigma o n}^{-}+i o\right)} \\
= & \left(i g_{S}\right)^{2} \frac{\theta\left(k_{1}^{+}-k_{1}^{\prime+}\right)}{\left(k_{1}^{+}-k_{1}^{\prime+}\right)} \frac{i}{\left(K^{-}-\frac{\vec{k}_{1 \perp}^{\prime 2}+m_{1}^{2}}{k_{1}^{\prime+}}-\frac{\left(\vec{K}_{\perp}-\vec{k}_{1 \perp}\right)^{2}+m_{2}^{2}}{K^{+}-k_{1}^{+}}-\frac{\left(\vec{k}_{1 \perp}^{\prime}-\vec{k}_{1 \perp}\right)^{2}+\mu^{2}}{k_{1}^{+}-k_{1}^{\prime+}}+i o\right)} \\
& +\left(i g_{S}\right)^{2} \frac{\theta\left(k_{1}^{\prime+}-k_{1}^{+}\right)}{\left(k_{1}^{\prime+}-k_{1}^{+}\right)} \frac{i}{\left(K^{-}-\frac{\vec{k}_{1 \perp}^{2}+m_{1}^{2}}{k_{1}^{+}}-\frac{\left(\vec{K}_{\perp}-\vec{k}_{1 \perp}^{\prime}\right)^{2}+m_{2}^{2}}{K^{+}-k_{1}^{\prime+}}-\frac{\left(\vec{k}_{1 \perp}^{\prime}-\vec{k}_{1 \perp}\right)^{2}+\mu^{2}}{k_{1}^{\prime+}-k_{1}^{+}}+i o\right)}
\end{aligned}
$$

\section{APPENDIX C: INTERACTION IN SECOND ORDER}

The interaction $w(k)$, defined by Eqs. (37) and (11) to second order in the driving term $V(K)$, is given by

$$
w(K) \simeq w^{(2)}(K)+w^{(4)}(K),
$$

where $w^{(2)}(K)$ is given by Eq. (B5) and

$$
w^{(4)}(K)=g_{0}(K)^{-1}\left|G_{0}(K) V(K) G_{0}(K) V(K) G_{0}(K)\right| g_{0}(K)^{-1}-g_{0}(K)^{-1}\left|G_{0}(K) V(K) \widetilde{G}_{0}(K) V(K) G_{0}(K)\right| g_{0}(K)^{-1}
$$

The second term in Eq. (C2) corresponds to the iteration of the interaction $w^{(2)}(K)$

$$
\begin{aligned}
& g_{0}(K)^{-1}\left|G_{0}(K) V(K) \widetilde{G}_{0}(K) V(K) G_{0}(K)\right| g_{0}(K)^{-1} \\
& \quad=g_{0}(K)^{-1}\left|G_{0}(K) V(K) G_{0}(K)\right| g_{0}(K)^{-1}\left|G_{0}(K) V(K) G_{0}(K)\right| g_{0}(K)^{-1} \\
& \quad=w^{(2)} g_{0}(K) w^{(2)}
\end{aligned}
$$

The matrix element of the operator $\left|G_{0}(K) V(K) G_{0}(K) V(K) G_{0}(K)\right|$ is 


$$
\begin{aligned}
& \left\langle k_{1}^{\prime+} \vec{k}_{1 \perp}^{\prime}\left\|G_{0}(K) V(K) G_{0}(K) V(K) G_{0}(K)\right\| k_{1}^{+} \vec{k}_{1 \perp}\right\rangle \\
& =\frac{\left(i g_{S}\right)^{4}}{2(2 \pi)^{6}} \int d k_{1}^{\prime-} d p_{1}^{-} d k_{1}^{-} d p_{1}^{+} d^{2} p_{1 \perp} \frac{1}{k_{1}^{\prime+}\left(K^{+}-k_{1}^{\prime+}\right)} \frac{1}{\left(k_{1}^{\prime-}-\frac{\vec{k}_{1 \perp}^{\prime 2}+m_{1}^{2}-i o}{k_{1}^{\prime+}}\right)} \\
& \times \frac{1}{\left(K^{-}-k_{1}^{\prime-}-\frac{\left(\vec{K}_{\perp}-\vec{k}_{1 \perp}^{\prime 2}\right)+m_{2}^{2}-i o}{K^{+}-k_{1}^{\prime+}}\right)} \frac{1}{\left(k_{1}^{\prime+}-p_{1}^{+}\right)} \frac{1}{\left(k_{1}^{\prime-}-p_{1}^{-}-\frac{\left(\vec{k}_{1 \perp}^{\prime}-\vec{p}_{1 \perp}\right)^{2}+\mu^{2}-i o}{k_{1}^{\prime+}-p_{1}^{+}}\right)} \\
& \times \frac{1}{p_{1}^{+}\left(K^{+}-p_{1}^{+}\right)} \frac{1}{\left(p_{1}^{-}-\frac{\vec{p}_{1 \perp}^{2}+m_{1}^{2}-i o}{p_{1}^{+}}\right)} \frac{1}{\left(K^{-}-p_{1}^{-}-\frac{\left(\vec{K}_{\perp}-\vec{p}_{1 \perp}\right)^{2}+m_{2}^{2}-i o}{K^{+}-p_{1}^{+}}\right)} \frac{1}{\left(p_{1}^{+}-k_{1}^{+}\right)} \\
& \times \frac{1}{\left(p_{1}^{-}-k_{1}^{-}-\frac{\left(\vec{p}_{1 \perp}-\vec{k}_{1 \perp}\right)^{2}+\mu^{2}-i o}{p_{1}^{+}-k_{1}^{+}}\right)} \frac{1}{k_{1}^{+}\left(K^{+}-k_{1}^{+}\right)} \frac{1}{\left(k_{1}^{-}-\frac{\vec{k}_{1 \perp}^{2}+m_{1}^{2}-i o}{k_{1}^{+}}\right)} \frac{1}{\left(K^{-}-k_{1}^{-}-\frac{\left(\vec{K}_{\perp}-\vec{k}_{1 \perp}\right)^{2}+m_{2}^{2}-i o}{K^{+}-k_{1}^{+}}\right)} .
\end{aligned}
$$

The on-energy-shell values of the light-front minus momentum in Eq. (C4) are given in Eq. (B4), and

$$
\begin{gathered}
p_{1 o n}^{-}=\frac{\vec{p}_{1 \perp}^{2}+m_{1}^{2}}{p_{1}^{+}}, \\
p_{2 o n}^{-}=\frac{\left(\vec{K}_{\perp}-\vec{p}_{1 \perp}\right)^{2}+m_{2}^{2}}{K^{+}-p_{1}^{+}} .
\end{gathered}
$$

The matrix element $\left\langle k_{1}^{\prime+} \vec{k}_{1 \perp}^{\prime}\left\|G_{0}(K) V(K) G_{0}(K) V(K) G_{0}(K)\right\| k_{1}^{+} \vec{k}_{1 \perp}\right\rangle$ is found by analytical integration in the light-front "energies" in Eq. (C4). To separate the intermediate four particle propagation, which occurs for $k_{1}^{\prime+}, p_{1}^{+}$, and $k_{1}^{+}$satisfying $0<k_{1}^{+}<p_{1}^{+}<k^{\prime+}<K^{+}$, the following factorization is necessary:

$$
\begin{aligned}
\frac{1}{K^{-}-} p_{1}^{-}-\frac{\left(\vec{K}_{\perp}-\vec{p}_{1 \perp}\right)^{2}+m_{2}^{2}-i o}{K^{+}-p_{1}^{+}} \times \frac{1}{p_{1}^{-}-k_{1}^{-}-\frac{\left(\vec{k}_{1 \perp}-\vec{p}_{1}\right)^{2}+\mu^{2}-i o}{p_{1}^{+}-k_{1}^{+}}} \\
=\frac{1}{K^{-}-k_{1}^{-}-\frac{\left(\vec{K}_{\perp}-\vec{p}_{1 \perp}\right)^{2}+m_{2}^{2}-i o}{K^{+}-p_{1}^{+}}-\frac{\left(\vec{k}_{1 \perp}-\vec{p}_{1 \perp}\right)^{2}+\mu^{2}-i o}{p_{1}^{+}-k_{1}^{+}}} \\
\quad \times\left[\frac{1}{K^{-}-p_{1}^{-}-\frac{\left(\vec{K}_{\perp}-\vec{p}_{1 \perp}\right)^{2}+m_{2}^{2}-i o}{K^{+}-p_{1}^{+}}}+\frac{1}{p_{1}^{-}-k_{1}^{-}-\frac{\left(\vec{k}_{1 \perp}-\vec{p}_{1 \perp}\right)^{2}+\mu^{2}-i o}{p_{1}^{+}-k_{1}^{+}}}\right] .
\end{aligned}
$$

After the Cauchy integration in the light-front "energies" the result for $\left\langle k_{1}^{\prime+} \vec{k}_{1 \perp}^{\prime}\left\|G_{0}(K) V(K) G_{0}(K) V(K) G_{0}(K)\right\| k_{1}^{+} \vec{k}_{1 \perp}\right\rangle$ in the region of $0<k_{1}^{+}<p_{1}^{+}<k_{1}^{\prime+}<K^{+}$, which is denoted by $\left\langle\left. k_{1}^{\prime+} \vec{k}_{1 \perp}^{\prime}|| G_{0}(K) V(K) G_{0}(K) V(K) G_{0}(K)\right|_{(a)} \mid k_{1}^{+} \vec{k}_{1 \perp}\right\rangle$, is given by 


$$
\begin{aligned}
& \left\langle k_{1}^{\prime+} \vec{k}_{1 \perp}^{\prime} \|\left. G_{0}(K) V(K) G_{0}(K) V(K) G_{0}(K)\right|_{(a)} \mid k_{1}^{+} \vec{k}_{1 \perp}\right\rangle \\
& =\frac{\left(i g_{S}\right)^{4}}{2(2 \pi)^{3}} \int d p_{1}^{+} d^{2} p_{1 \perp} \frac{\theta\left(k_{1}^{\prime+}\right) \theta\left(K^{+}-k_{1}^{\prime+}\right)}{k_{1}^{\prime+}\left(K^{+}-k_{1}^{\prime+}\right)} \frac{i}{K^{-}-\frac{\vec{k}_{1 \perp}^{\prime 2}+m_{1}^{2}}{k_{1}^{\prime+}}-\frac{\left(\vec{K}_{\perp}-\vec{k}_{1 \perp}^{\prime}\right)^{2}+m_{2}^{2}}{K^{+}-k_{1}^{\prime+}}+i o} \\
& \quad \times\left[F^{\prime}(K)+F^{\prime \prime}(K)\right] \frac{\theta\left(k_{1}^{+}\right) \theta\left(K^{+}-k_{1}^{+}\right)}{k_{1}^{+}\left(K^{+}-k_{1}^{+}\right)} \frac{i}{K^{-}-\frac{\vec{k}_{1 \perp}^{2}+m_{1}^{2}}{k_{1}^{+}}-\frac{\left(\vec{K}_{\perp}-\vec{k}_{1 \perp}\right)^{2}+m_{2}^{2}}{K^{+}-k_{1}^{+}}+i o},
\end{aligned}
$$

with

$$
\begin{aligned}
& F^{\prime}(K)=\frac{\theta\left(k_{1}^{\prime+}-p_{1}^{+}\right)}{\left(k_{1}^{\prime+}-p_{1}^{+}\right)} \frac{i}{K^{-}-\frac{\vec{p}_{1 \perp}^{2}+m_{1}^{2}}{p_{1}^{+}}-\frac{\left(\vec{K}_{\perp}-\vec{k}_{1 \perp}^{\prime}\right)^{2}+m_{2}^{2}}{K^{+}-k_{1}^{\prime+}}-\frac{\left(\vec{k}_{1 \perp}^{\prime}-\vec{p}_{1 \perp}\right)^{2}+\mu^{2}}{k_{1}^{\prime+}-p_{1}^{+}}+i o} \\
& \times \frac{\theta\left(p_{1}^{+}\right) \theta\left(K^{+}-p_{1}^{+}\right)}{p_{1}^{+}\left(K^{+}-p_{1}^{+}\right)} \frac{i}{K^{-}-\frac{\vec{p}_{1 \perp}^{2}+m_{1}^{2}}{p_{1}^{+}}-\frac{\left(\vec{K}_{\perp}-\vec{p}_{1 \perp}\right)^{2}+m_{2}^{2}}{K^{+}-p_{1}^{+}}+i o} \frac{\theta\left(p_{1}^{+}-k_{1}^{+}\right)}{\left(p_{1}^{+}-k_{1}^{+}\right)} \\
& \times \frac{i}{K^{-}-\frac{\vec{k}_{1 \perp}^{2}+m_{1}^{2}}{k_{1}^{+}}-\frac{\left(\vec{K}_{\perp}-\vec{p}_{1 \perp}\right)^{2}+m_{2}^{2}}{K^{+}-p_{1}^{+}}-\frac{\left(\vec{p}_{1 \perp}-\vec{k}_{1 \perp}\right)^{2}+\mu^{2}}{p_{1}^{+}-k_{1}^{+}}+i o} ; \\
& F^{\prime \prime}(K)=\frac{\theta\left(k_{1}^{\prime+}-p_{1}^{+}\right)}{\left(k_{1}^{\prime+}-p_{1}^{+}\right)} \frac{i}{K^{-}-\frac{\vec{p}_{1 \perp}^{2}+m_{1}^{2}}{p_{1}^{+}}-\frac{\left(\vec{K}_{\perp}-\vec{k}_{1 \perp}^{\prime}\right)^{2}+m_{2}^{2}}{K^{+}-k_{1}^{\prime+}}-\frac{\left(\vec{k}_{1 \perp}^{\prime}-\vec{p}_{1 \perp}\right)^{2}+\mu^{2}}{k_{1}^{\prime+}-p_{1}^{+}}+i o} \\
& \times \frac{i}{K^{-}-\frac{\vec{k}_{1 \perp}^{2}+m_{1}^{2}}{k_{1}^{+}}-\frac{\left(\vec{K}_{\perp}-\vec{k}_{1 \perp}^{\prime}\right)^{2}+m_{2}^{2}}{K^{+}-k_{1}^{\prime+}}-\frac{\left(\vec{k}_{1 \perp}^{\prime}-\vec{p}_{1 \perp}\right)^{2}+\mu^{2}}{k_{1}^{\prime+}-p_{1}^{+}}-\frac{\left(\vec{p}_{1 \perp}-\vec{k}_{1 \perp}\right)^{2}+\mu^{2}}{p_{1}^{+}-k_{1}^{+}}+i o} \\
& \times \frac{\theta\left(p_{1}^{+}-k_{1}^{+}\right)}{\left(p_{1}^{+}-k_{1}^{+}\right)} \frac{i}{K^{-}-\frac{\vec{k}_{1 \perp}^{2}+m_{1}^{2}}{k_{1}^{+}}-\frac{\left(\vec{K}_{\perp}-\vec{p}_{1 \perp}\right)^{2}+m_{2}^{2}}{K^{+}-p_{1}^{+}}-\frac{\left(\vec{p}_{1 \perp}-\vec{k}_{1 \perp}\right)^{2}+\mu^{2}}{p_{1}^{+}-k_{1}^{+}}+i o} .
\end{aligned}
$$

The part of the propagator given by Eq. (C7) contains the virtual light-front propagation of intermediate states with up to four particles. The function $F^{\prime}$ contains only intermediate states up to three particles and is two-body reducible. It will eventually be canceled by the corresponding piece in the second term in Eq. (C2). The function $F^{\prime \prime}$ has one intermediate state in which the four-particle propagator can be recognized as the middle piece of Eq. (C9). The other possibility that includes up to four particles in the intermediate state propagation is given by $0<k_{1}^{\prime+}<p_{1}^{+}<k_{1}^{+}<K^{+}$. To obtain this part, we perform the transformation $k_{1}^{\prime} \leftrightarrow k_{1}$ in Eq. (C7).

The contribution of the region given by $0<p_{1}^{+}<k_{1}^{+}<K^{+}$and $0<p_{1}^{+}<k_{1}^{\prime+}<K^{+}$to the matrix element $\left\langle k_{1}^{\prime+} \vec{k}_{1 \perp}^{\prime} \| G_{0}(K) V(K) G_{0}(K) V(K) G_{0}(K)|| k_{1}^{+} \vec{k}_{1 \perp}\right\rangle$ is denoted by $\left\langle\left. k_{1}^{\prime+} \vec{k}_{1 \perp}^{\prime}|| G_{0}(K) V(K) G_{0}(K) V(K) G_{0}(K)\right|_{(b)} \mid k_{1}^{+} \vec{k}_{1 \perp}\right\rangle$. It 
contains only up to three-particle intermediate states and is two-body reducible. Consequently, it will be canceled by the corresponding piece of the second term in Eq. (C2). It is given by

$$
\begin{aligned}
\left\langle\left. k_{1}^{\prime+} \vec{k}_{1 \perp}^{\prime}|| G_{0}(K) V(K) G_{0}(K) V(K) G_{0}(K)\right|_{(b)} \mid k_{1}^{+} \vec{k}_{1 \perp}\right\rangle & =\frac{\left(i g_{S}\right)^{4}}{2(2 \pi)^{3}} \int d p_{1}^{+} d^{2} p_{1 \perp} \frac{\theta\left(k_{1}^{\prime+}\right) \theta\left(K^{+}-k_{1}^{\prime+}\right)}{k_{1}^{\prime+}\left(K^{+}-k_{1}^{+}\right)} \frac{i}{K^{-}-\frac{\vec{k}_{1 \perp}^{\prime 2}+m_{1}^{2}}{k_{1}^{\prime+}}-\frac{\left(\vec{K}_{\perp}-k_{1 \perp}^{\prime}\right)^{2}+m_{2}^{2}}{K^{+}-k_{1}^{\prime+}}+i o} \frac{\theta\left(k_{1}^{\prime+}-p_{1}^{+}\right)}{\left(k_{1}^{\prime+}-p_{1}^{+}\right)} \\
& \times \frac{\theta\left(p_{1}^{+}\right) \theta\left(K^{+}-p_{1}^{+}\right)}{p_{1}^{+}\left(K^{+}-p_{1}^{+}\right)} \\
& \times \frac{K^{-}-\frac{\vec{p}_{1 \perp}^{2}+m_{1}^{2}}{p_{1}^{+}}-\frac{\left(\vec{K}_{\perp}-\vec{k}_{1 \perp}^{\prime}\right)^{2}+m_{2}^{2}}{K^{+}-k_{1}^{\prime+}}-\frac{\left(\vec{k}_{1 \perp}^{\prime}-\vec{p}_{1 \perp}\right)^{2}+\mu^{2}}{k_{1}^{\prime+}-p_{1}^{+}}+i o}{i} \frac{\theta\left(k_{1}^{+}-p_{1}^{+}\right)}{\left(k_{1}^{+}-p_{1}^{+}\right)} \\
& K^{-}-\frac{\vec{p}_{1 \perp}^{2}+m_{1}^{2}}{p_{1}^{+}}-\frac{\left(\vec{K}_{\perp}-\vec{p}_{1 \perp}\right)^{2}+m_{2}^{2}}{K^{+}-p_{1}^{+}}+i o \\
& \times \frac{\vec{p}^{2}}{K^{-}-\frac{p_{1 \perp}+m_{1}^{2}}{p_{1}^{+}}-\frac{\left(\vec{K}_{\perp}-\vec{k}_{1 \perp}\right)^{2}+m_{2}^{2}}{K^{+}-k_{1}^{+}}-\frac{\left(\vec{k}_{1 \perp}-\vec{p}_{1 \perp}\right)^{2}+\mu^{2}}{k_{1}^{+}-p_{1}^{+}}+i o} \\
& \times \frac{\theta\left(k_{1}^{+}\right) \theta\left(K^{+}-k_{1}^{+}\right)}{k_{1}^{+}\left(K^{+}-k_{1}^{+}\right)} \frac{K^{-}-\frac{\vec{k}_{1 \perp}^{2}+m_{1}^{2}}{k_{1}^{+}}-\frac{\left(\vec{K}_{\perp}-\vec{k}_{1 \perp}\right)^{2}+m_{2}^{2}}{K^{+}-k_{1}^{+}}}{}
\end{aligned}
$$

For the momentum region satisfying $0<k_{1}^{\prime+}<p_{1}^{+}<K^{+}$and $0<k_{1}^{+}<p_{1}^{+}<K^{+}$, the contribution to the matrix element $\left\langle k_{1}^{\prime+} \vec{k}_{1 \perp}^{\prime}\left\|G_{0}(K) V(K) G_{0}(K) V(K) G_{0}(K)\right\| k_{1}^{+} \vec{k}_{1 \perp}\right\rangle$ can be obtained from Eq. (C10) by performing the following transformation on the kinematical momentum: $k_{1}^{\prime} \leftrightarrow K-k_{1}^{\prime}, k_{1} \leftrightarrow K-k_{1}$ and $m_{1} \leftrightarrow m_{2}$. From Eqs. (C9) and (C10), the following result is obtained:

$$
\begin{aligned}
\left\langle k_{1}^{\prime+} \vec{k}_{1 \perp}^{\prime}|| G_{0}(K) V(K) G_{0}(K) V(K) G_{0}(K)|| k_{1}^{+} \vec{k}_{1 \perp}\right\rangle \\
=\left(\left\langle\left. k_{1}^{\prime+} \vec{k}_{1 \perp}^{\prime}|| G_{0}(K) V(K) G_{0}(K) V(K) G_{0}(K)\right|_{(a)} \mid k_{1}^{+} \vec{k}_{1 \perp}\right\rangle+\left[k_{1}^{\prime} \leftrightarrow k_{1}\right]\right) \\
\quad+\left(\left\langle k_{1}^{\prime+} \vec{k}_{1 \perp}^{\prime}\left|G_{0}(K) V(K) G_{0}(K) V(K) G_{0}(K)\right|_{(b)} \mid k_{1}^{+} \vec{k}_{1 \perp}\right\rangle+\left[k_{1}^{\prime} \leftrightarrow K-k_{1}^{\prime}, k_{1} \leftrightarrow K-k_{1}, m_{1} \leftrightarrow m_{2}\right]\right) .
\end{aligned}
$$

The subtraction of the iterated first order driving term in Eq. (C2) cancels the corresponding terms in Eq. (C11) such that the matrix element $\left\langle k_{1}^{\prime+} \vec{k}_{1 \perp}^{\prime}\left|w^{(4)}(K)\right| k_{1}^{+} \vec{k}_{1 \perp}\right\rangle$ is two-body irreducible with a global four-body propagation. It is obtained from Eqs. (C7), (C9), and (C2) as

$$
\begin{aligned}
\left\langle k_{1}^{\prime+} \vec{k}_{1 \perp}^{\prime}\left|w^{(4)}(K)\right| k_{1}^{+} \vec{k}_{1 \perp}\right\rangle= & \frac{\left(i g_{S}\right)^{4}}{2(2 \pi)^{3}} \int d p_{1}^{+} d^{2} p_{1 \perp} \frac{\theta\left(k_{1}^{\prime+}-p_{1}^{+}\right)}{\left(k_{1}^{\prime+}-p_{1}^{+}\right)} \frac{\theta\left(p_{1}^{+}\right)}{p_{1}^{+}} \frac{\theta\left(K^{+}-p_{1}^{+}\right)}{K^{+}-p_{1}^{+}} \\
& \times \frac{i}{K^{-}-\frac{\vec{p}_{1 \perp}^{2}+m_{1}^{2}}{p_{1}^{+}}-\frac{\left(\vec{K}_{\perp}-\vec{k}_{1 \perp}^{\prime}\right)^{2}+m_{2}^{2}}{K^{+}-k_{1}^{\prime+}}-\frac{\left(\vec{k}_{1 \perp}^{\prime}-\vec{p}_{1 \perp}\right)^{2}+\mu^{2}}{k_{1}^{\prime+}-p_{1}^{+}}+i o}
\end{aligned}
$$




$$
\begin{aligned}
& \times \frac{\vec{k}_{1 \perp}^{2}+m_{1}^{2}}{k_{1}^{+}}-\frac{\left(\vec{K}_{\perp}-\vec{k}_{1 \perp}^{\prime}\right)^{2}+m_{2}^{2}}{K^{+}-k_{1}^{\prime+}}-\frac{\left(\vec{k}_{1 \perp}^{\prime}-\vec{p}_{1 \perp}\right)^{2}+\mu^{2}}{k_{1}^{\prime+}-p_{1}^{+}}-\frac{\left(\vec{p}_{1 \perp}-\vec{k}_{1 \perp}\right)^{2}+\mu^{2}}{p_{1}^{+}-k_{1}^{+}}+i o \\
& \times \frac{\theta\left(p_{1}^{+}-k_{1}^{+}\right)}{\left(p_{1}^{+}-k_{1}^{+}\right)} \frac{i}{K^{-}-\frac{\vec{k}_{1 \perp}^{2}+m_{1}^{2}}{k_{1}^{+}}-\frac{\left(\vec{K}_{\perp}-\vec{p}_{1 \perp}\right)^{2}+m_{2}^{2}}{K^{+}-p_{1}^{+}}-\frac{\left(\vec{p}_{1 \perp}-\vec{k}_{1 \perp}\right)^{2}+\mu^{2}}{p_{1}^{+}-k_{1}^{+}}+i o}+\left[k_{1}^{\prime} \leftrightarrow k_{1}\right] .
\end{aligned}
$$

\section{APPENDIX D: INTEGRAL EQUATION FOR THE BOUND-STATE}

In the approximation considered, the vertex function satisfies an integral equation with the kernel containing two parts, one corresponding to Eq. (B5) and the other to Eq. (C12). The plus momentum are rescaled by $K^{+}$, such that the momentum fractions $x=k_{1}^{+} / K^{+}, y=k_{1}^{\prime+} / K^{+}$, and $z=p_{1}^{+} / K^{+}$, are used. The notation $\left\langle k_{1}^{\prime+} \vec{k}_{1 \perp}^{\prime} \mid \gamma_{B}\right\rangle \equiv \gamma_{B}\left(y, \vec{k}_{1 \perp}^{\prime}\right)$ is introduced. The homogeneous integral equation for the light-front vertex function is evaluated in the center of mass system

$$
\gamma_{B}\left(y, \vec{k}_{1 \perp}^{\prime}\right)=\frac{1}{(2 \pi)^{3}} \int \frac{d^{2} k_{1 \perp} d x}{2 x(1-x)} \frac{\mathcal{K}^{(2)}\left(y, \vec{k}_{1 \perp}^{\prime} ; x, \vec{k}_{1 \perp}\right)+\mathcal{K}^{(4)}\left(y, \vec{k}_{1 \perp}^{\prime} ; x, \vec{k}_{1 \perp}\right)}{M_{B}^{2}-M_{0}^{2}} \gamma_{B}\left(x, \vec{k}_{1 \perp}\right),
$$

where the free two-body mass is $M_{0}^{2}=\left(\vec{k}_{1 \perp}^{2}+m^{2}\right) / x(1-x)$ and $0<x<1$.

The part of the kernel which has only the propagation of virtual three particles states foward in the light-front time is obtained from Eq. (B5),

$$
\mathcal{K}^{(2)}\left(y, \vec{k}_{1 \perp}^{\prime} ; x, \vec{k}_{1 \perp}\right)=g_{S}^{2} \frac{\theta(x-y)}{(x-y)\left(M_{B}^{2}-\frac{\vec{k}_{1 \perp}^{\prime 2}+m^{2}}{y}-\frac{\vec{k}_{1 \perp}^{2}+m^{2}}{1-x}-\frac{\left(\vec{k}_{1 \perp}^{\prime}-\vec{k}_{1 \perp}\right)^{2}+\mu^{2}}{x-y}\right)}+\left[x \leftrightarrow y, \vec{k}_{1 \perp}^{\prime} \leftrightarrow \vec{k}_{1 \perp}\right]
$$

Equation (D1) with the effective interaction given by Eq. (D2) corresponds to the Weinberg equation derived from the BSE in the infinitum momentum frame [20]. It has also been solved in Ref. [21] and in Ref. [22] including self-energy correction. The equivalent equation for fermions has been discussed in Ref. [23].

The contribution to the kernel from the virtual four-body propagation is obtained from Eq. (C12),

$$
\begin{aligned}
\mathcal{K}^{(4)}\left(y, \vec{k}_{1 \perp}^{\prime} ; x, \vec{k}_{1 \perp}\right)= & \frac{g_{S}^{4}}{(2 \pi)^{3}} \int \frac{d^{2} p_{1 \perp} d z}{2 z(1-z)(z-x)(z-y)} \frac{\theta(z-y) \theta(x-z)}{\left.M_{B}^{2}-\frac{\vec{k}_{1 \perp}^{\prime 2}+m^{2}}{y}-\frac{\vec{p}_{1 \perp}^{2}+m^{2}}{1-z}-\frac{\left(\vec{k}_{1 \perp}^{\prime}-\vec{p}_{1 \perp}\right)^{2}+\mu^{2}}{z-y}\right)} \\
& \times \frac{1}{\left(M_{B}^{2}-\frac{\vec{k}_{1 \perp}^{\prime 2}+m^{2}}{y}-\frac{\vec{k}_{1 \perp}^{2}+m^{2}}{1-x}-\frac{\left(\vec{k}_{1 \perp}^{\prime}-\vec{p}_{1 \perp}\right)^{2}+\mu^{2}}{z-y}-\frac{\left(\vec{p}_{1 \perp}-\vec{k}_{1 \perp}\right)^{2}+\mu^{2}}{x-z}\right)} \\
& \times \frac{1}{\left(M_{B}^{2}-\frac{\vec{p}_{1 \perp}^{2}+m^{2}}{z}-\frac{\vec{k}_{1 \perp}^{2}+m^{2}}{1-x}-\frac{\left(\vec{p}_{1 \perp}-\vec{k}_{1 \perp}\right)^{2}+\mu^{2}}{x-z}+\left[x \leftrightarrow y, \vec{k}_{1 \perp} \leftrightarrow \vec{k}_{1 \perp}^{\prime}\right] .\right.}
\end{aligned}
$$

Equations (D1)-(D3) are easily recognized to be covariant under kinematical light-front boosts. However, the covariance of the four-dimensional wave function (41) is certainly lost by a finite expansion of $W(K)$ in Eq. (11) and the use of the corresponding $w(K)$ while covariance continues to hold for the solution of Eq. (11).

[1] E. E. Salpeter and H. A. Bethe, Phys. Rev. 84, 1232 (1951).

[2] J. Fleischer and J. A. Tjon, Nucl. Phys. B84, 375 (1975); Phys. Rev. D 15, 2537 (1977); 21, 87 (1980).

[3] T. Nieuwenhuis and J. A. Tjon, Few-Body Syst. 21, 167
(1996); T. Nieuwenhuis and J. A. Tjon, Phys. Rev. Lett. 77, 814 (1996).

[4] M. J. Zuilhof and J. A. Tjon, Phys. Rev. C 22, 2369 (1980).

[5] K. Kusaka, K. Simpson, and A. G. Willians, Phys. Rev. D 56, 
5071 (1997).

[6] F. Gross, Phys. Rev. 186, 1448 (1969); Phys. Rev. C 26, 2203 (1982).

[7] V. Pascalutsa and J. A. Tjon, Phys. Lett. B 435, 245 (1998).

[8] M. G. Fuda, Phys. Rev. C 51, 23 (1995); 52, 1260 (1995).

[9] P. G. Blunden, M. Burkardt, and G. A. Miller, Phys. Rev. C 59, 2998 (1999); 60, 055211 (1999).

[10] G. A. Miller and R. Machleidt, Phys. Lett. B 455, 19 (1999); Phys. Rev. C 60, 035202 (1999).

[11] J. R. Cooke, G. A. Miller, and D. R. Phillips, "Restoration of Rotational Invariance of Bound-States on the Light-Front,", nucl-th/9910013.

[12] R. M. Woloshyn and A. D. Jackson, Nucl. Phys. B64, 269 (1973).

[13] C. Itzykson and J.-B. Zuber, Quantum Field Theory (McGrawHill, New York, 1985).

[14] P. A. M. Dirac, Rev. Mod. Phys. 21, 392 (1949).
[15] S. J. Brodsky, H.-C. Pauli, and S. S. Pinsky, Phys. Rep. 301, 299 (1998).

[16] N. C. J. Schoonderwoerd, B. L. G. Bakker, and V. A. Karmanov, Phys. Rev. C 58, 3093 (1998).

[17] L. L. Frankfurt and M. I. Strikman, Nucl. Phys. B148, 107 (1979); Phys. Rep. 76, 215 (1981).

[18] T. Frederico and R.-W. Schulze, Phys. Rev. C 54, 2201 (1996).

[19] J. Carbonell, B. Desplanques, V. A. Karmanov, and J. F. Mathiot, Phys. Rep. 300, 215 (1998).

[20] S. Weinberg, Phys. Rev. 150, 1313 (1966).

[21] C.-R. Ji and R. J. Furnstahl, Phys. Lett. B 167, 11 (1986); C.-R. Ji, ibid. 167, 16 (1986).

[22] C.-R. Ji, Phys. Lett. B 322, 389 (1994).

[23] R. J. Perry, A. Harindranath, and K. G. Wilson, Phys. Rev. Lett. 65, 2959 (1990); S. Glasek, A. Harindranath, S. Pinsky, J. Shigemitsu, and K. G. Wilson, Phys. Rev. D 47, 1599 (1993). 